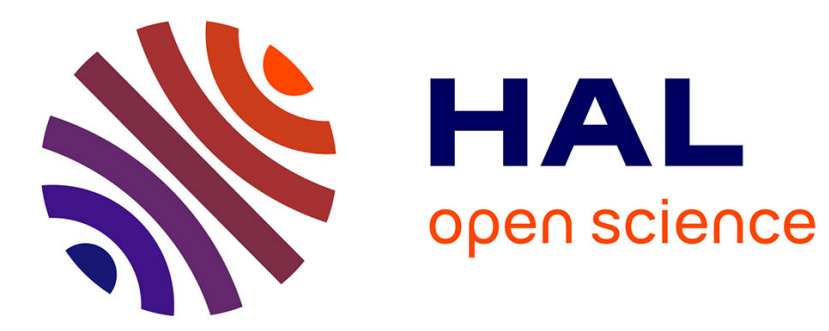

\title{
Crossover between Re-Nucleation and Dendritic Growth in Electrodeposition without Supporting Electrolyte
}

\author{
Chams Kharbachi, Théo Tzedakis, Fabien Chauvet
}

\section{To cite this version:}

Chams Kharbachi, Théo Tzedakis, Fabien Chauvet. Crossover between Re-Nucleation and Dendritic Growth in Electrodeposition without Supporting Electrolyte. Journal of The Electrochemical Society, 2021, 168 (7), pp.072507. 10.1149/1945-7111/ac15bb . hal-03332007

\section{HAL Id: hal-03332007 https://hal.science/hal-03332007}

Submitted on 2 Sep 2021

HAL is a multi-disciplinary open access archive for the deposit and dissemination of scientific research documents, whether they are published or not. The documents may come from teaching and research institutions in France or abroad, or from public or private research centers.
L'archive ouverte pluridisciplinaire HAL, est destinée au dépôt et à la diffusion de documents scientifiques de niveau recherche, publiés ou non, émanant des établissements d'enseignement et de recherche français ou étrangers, des laboratoires publics ou privés. 




Open Archive Toulouse Archive Ouverte

OATAO is an open access repository that collects the work of Toulouse researchers and makes it freely available over the web where possible

This is an author's version published in: http://oatao.univ-toulouse.fr/ 28215

Official URL : https://doi.org/10.1149/1945-7111/ac15bb

\section{To cite this version:}

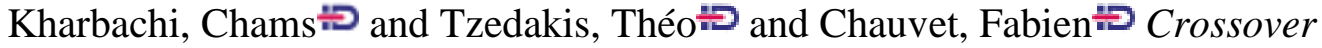
between Re-Nucleation and Dendritic Growth in Electrodeposition without Supporting Electrolyte. (2021) Journal of The Electrochemical Society (JES), 168 (7). ISSN 0013-4651

Any correspondence concerning this service should be sent to the repository administrator: tech-oatao@listes-diff.inp-toulouse.fr 


\title{
Crossover between Re-Nucleation and Dendritic Growth in Electrodeposition without Supporting Electrolyte
}

\author{
Chams Kharbachi, Théo Tzedakis, and Fabien Chauvet $^{*, z_{(1)}}$ \\ Laboratoire de Génie Chimique, Université de Toulouse, CNRS, INPT, UPS, Toulouse, France
}

\begin{abstract}
This work deals with the formation of dendritic structures by electrodeposition of $\mathrm{Cu}^{2+}$ and $\mathrm{Ag}^{+}$without supporting electrolyte in Hele-Shaw cells. The transition between the two main patterns, ramified branches and dendrites, is specifically addressed at the scale of branch microstructure using careful SEM observations. Ramified branches, composed only of grain assemblies, are obtained at low current densities because of a re-nucleation process induced by space charge dynamics (Fleury, Nature, 1997). For current densities higher than a given threshold, ramified branches are also formed by re-nucleation but another growth mode, the dendritic growth, is also observed while, at the macro-scale, the pattern remains fractal and isotropic. This shows that 1) pattern transition originates from a morphological transition at microstructure scale and 2) the re-nucleation process enables a freedom in local growth direction allowing the pattern to be fractal at the macro-scale. The onset of the dendritic growth mode, from shape instability of the grains, is considered with Mullins \& Sekerka model. This latter disagrees with the observations by predicting that the grains are always unstable. It is proposed that the space charge plays a key role by controlling the shape stability and thus the transition between the two growth modes.

(C) 2021 The Author(s). Published on behalf of The Electrochemical Society by IOP Publishing Limited. This is an open access article distributed under the terms of the Creative Commons Attribution 4.0 License (CC BY, http://creativecommons.org/licenses/ by/4.0/), which permits unrestricted reuse of the work in any medium, provided the original work is properly cited. [DOI: 10.1149/ $1945-7111 / \mathrm{ac} 15 \mathrm{bb}]$
\end{abstract}

Manuscript submitted June 10, 2021; revised manuscript received July 12, 2021. Published July 30, 2021.

The electrodeposition of a metal is known to lead to the formation of ramified structures commonly called dendrites. Depending on the application, dendrites must be avoided, as in $\mathrm{Li}$ batteries $^{1-3}$ and electroplating, ${ }^{4}$ or they can be advantageously exploited for their enhanced surface which gives them catalytic, ${ }^{5,6}$ optical $^{7,8}$ and wetting ${ }^{9,10}$ properties. Understanding and controlling the electrochemical formation of these ramified and dendritic structures is therefore of major interest in several fields such as energy, chemical sensing and coating of surfaces.

Among the possible configurations, the formation of ramified and dendritic structures in transparent Hele-Shaw cells, in absence of supporting electrolyte, is a model situation well suited for conducting fundamental studies. This leads to the fast growth of twodimensional patterns which are visible to the naked eye. The reader should be aware that the formation of such structures is explained by the fact that no supporting electrolyte is used; otherwise, the reduction of the solvent (water) takes place rapidly and this leads to the formation of hydrogen bubbles which disturb, or even prevent, the growth of ramified or dendritic structures; ${ }^{11}$ note that, here, in the considered situations, the applied current is always higher than the limiting current. ${ }^{12}$ As a consequence, to avoid the separation of the charge carriers in the solution (anions and cations of the dissolved metal salt), i.e. the deviation from electroneutrality, the growth front (cathode) must move towards the anode at the same velocity as that of the anions whose the movement is driven by electromigration away from the cathode. ${ }^{3,12-14}$ Since this velocity is typically high $\left(\sim 10 \mu \mathrm{m} \mathrm{s}^{-1}\right)$, the deposit density is very low that leads to ramified structures; ${ }^{13,15}$ in addition, note that if a supporting electrolyte is used, this growth mechanism is suppressed because the presence of the inert ions of the supporting electrolyte prevents charge separation. The resulting system of interest here is therefore an electrochemical cell with a high electric resistance (no supporting electrolyte and thin cell). The corresponding ohmic drop induces a high cell voltage which can typically reach several volts.

During the 80's and 90's, the electrochemical growth of these structures was used as a model situation for theoretical studies on pattern formation in connection with Diffusion Limited Aggregation (DLA) patterns. ${ }^{16-23}$ Indeed, even if the cations and anions are transported by electromigration in addition to diffusion, the system of equations governing the growth phenomenon can be converted

*Electrochemical Society Member.

${ }^{\mathrm{z}}$ E-mail: fabien.chauvet@univ-tlse3.fr into an usual diffusion problem, assuming electroneutrality in the whole electrolyte domain (transport in a binary electrolyte). ${ }^{15,24} \mathrm{At}$ the macro-scale, the concentration field (of cations and anions) follows a diffusion-limited dynamics, as verified experimentally by both interferometric measurements ${ }^{25}$ and morphological analyzes of the electrodeposits (fractal property). ${ }^{26}$

From the previous works it appeared that, depending on the operating parameters (imposed electric current, concentration of metal salt, etc.) and on the nature of the metal, two main kinds of patterns are obtained at the macro-scale (length scales $>\sim 10 \mu \mathrm{m}$ ): ramified branches and dendrites. ${ }^{18,27,28}$

The ramified branches are fractal because the growth process is mainly diffusion-limited without stabilizing effects (surface energy and kinetics) at the macro-scale. In other words, the growth front is unconditionally unstable (any protrusion is amplified), that results in a random selection of growth directions and a fractal pattern on a given range of length scales. ${ }^{19,26}$ The pattern of ramified branches can be of two types: fractal-like and Dense Branching (DB). A fractal-like pattern is obtained in the limit of low growth velocities (low applied currents). ${ }^{26}$ Under this condition, the growth process is then close to the Laplacian growth model giving rise to a fractal deposit with an infinite upper cut-off length scale, i.e. a DLA pattern. $^{19,29}$ The DB patterns are obtained for higher and finite growth velocities. The branches then grow behind an almost flat growth front. The DB patterns are also fractal but on a limited range of length scales with a finite upper bound due to the stabilization of the growth front by a finite diffusion length. ${ }^{13,15,30-32}$ As resumed by Léger et al. 2000, ${ }^{15}$ the transition between fractal-like and DB patterns is still not well understood. Indeed, this is difficult to know if this transition is intrinsically related to the diffusion, because of the presence of interfering effects such as several kinds of convection (natural convection, ${ }^{2,33}$ electroconvection ${ }^{34}$ and electro-osmosis ${ }^{22}$ ) or the electrical resistance of the branches themselves. ${ }^{35}$

Dendritic deposits are typically observed for even higher applied currents than for DB patterns and mainly with zinc. ${ }^{17,98}$ Note that here, the word "dendrite" is used to name a single crystal which has been formed by an out-of-equilibrium growth with anisotropy effects (surface energy and/or kinetics). This results in a crystal shape with well-ordered geometry, such as a main arm with different levels of side branching oriented with a constant angle. This differs from the random and isotropic shape of the ramified branches as sketched in Fig. 1. Grier et al. $1986^{17}$ explain the appearance of the dendrites (at the macro-scale) by an exacerbated difference in growth rate, as a 


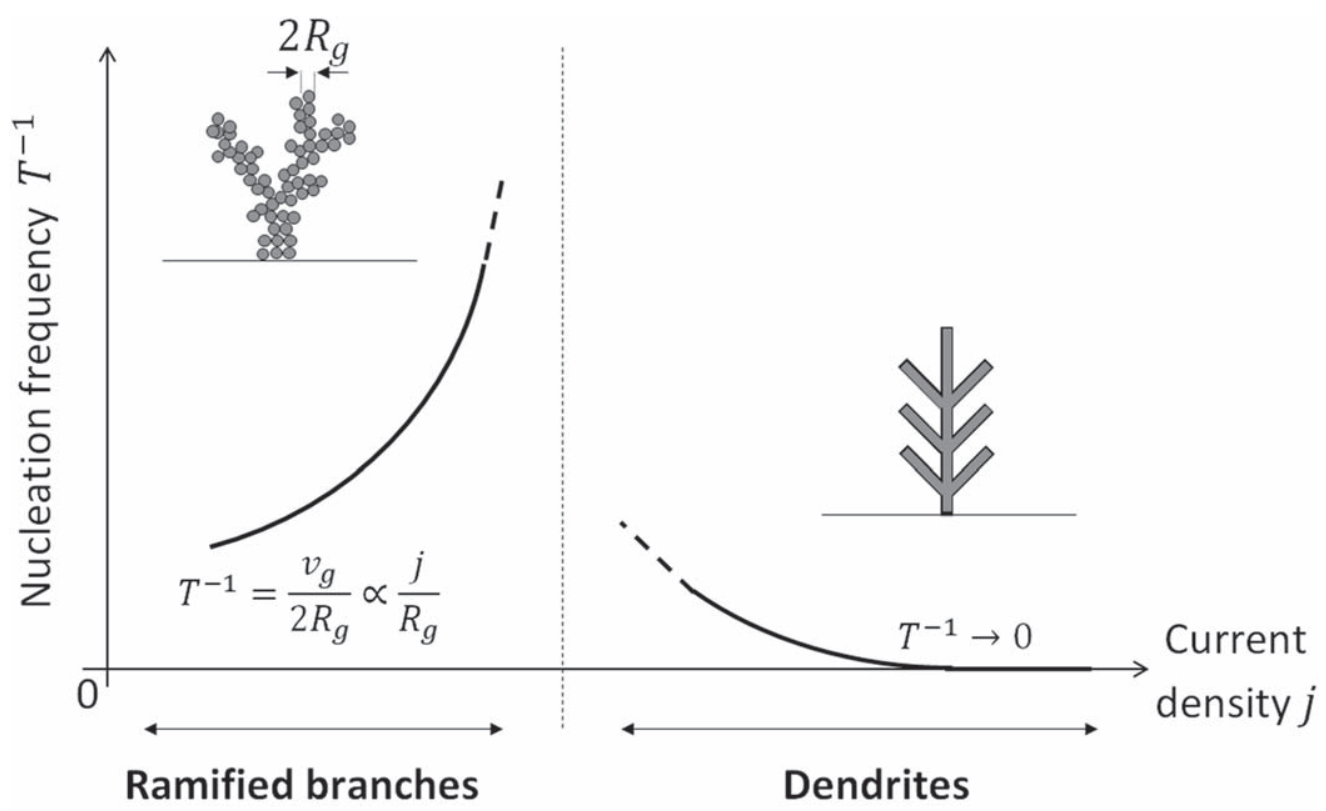

\section{Nucleation \& growth Growth (no re-nucleation)}

Figure 1. Illustration of the dependence of the nucleation frequency $T^{-1}$ on the applied current density $j$, when the growth regime changes from re-nucleation process (ramified branches) to pure growth without re-nucleation process (dendrites).

function of the orientation, when the current is high. In other terms, this corresponds to the out-of-equilibrium growth of a single anisotropic crystal. Obviously, the dendrite shape depends on the crystal structure of the metal, as noted by Grier et al. $1986^{17}$ by comparing copper and zinc deposits on the macro-scale. There is a significant difference in microstructure between dendritic deposits and ramified branches, as revealed by X-ray diffraction. ${ }^{17}$ As expected, the X-ray diffraction pattern is anisotropic for dendrites (single crystals) but isotropic for ramified branches. This suggests a polycrystalline structure for ramified branches (Fig. 1). Note that since the pioneer work of Barton and Bockris, ${ }^{36}$ dealing with the propagation of the dendrite tip across the electrolyte, the electrochemical dendritic growth was extensively studied focusing on the coupling between kinetics, mass transport and surface energy in dedicated experiments where the overpotential is applied instead of the current. ${ }^{37,38}$ Furthermore, a supporting electrolyte is generally used and the current employed is lower than the limiting current (a situation encountered in batteries), so this situation is different from the one of interest here. The predictions of this standard theory of the electrochemical dendritic growth have not yet been compared with the dynamics of dendritic deposits obtained in Hele-Shaw cells without the use of supporting electrolyte.

Very little work has focused on the micro/nano structure of the ramified branches. One reason is the difficult recovery of these fragile branches without damaging them. Using a cell, in which the inner bottom wall is covered with a non-percolating (non-conductive) gold coating (metallization by vapor deposition), Fleury ${ }^{39}$ succeeded in forming copper ramified branches that adhere to this wall. SEM observations of these plates show that the branches consist of small metallic (non-dendritic) crystals or grains whose size could be lower than $100 \mathrm{~nm}$. To date, Fleury proposes the most advanced interpretation and modeling for the building of this structure during branch growth: in order to match the average growth velocity to the velocity of anions, new grains must periodically nucleate and grow at the top of the branches. This principle is based on the fact that, assuming a given grain grows at a constant rate, after a given duration, the local growth velocity (on the grain surface) becomes lower than the velocity of the anions. ${ }^{39}$ This leads, in the vicinity of the metal surface, to the formation of a space charge region where the electroneutrality is not respected. ${ }^{14}$ Furthermore, at the surface of the growing metal, the electrolyte depletion induces the increase (even divergence) of both the electric field $E_{s}$ and the cell voltage. ${ }^{40,41}$ When exceeds a given threshold, a new grain nucleates and grows and the cycle restarts. ${ }^{39}$ Fleury showed that the grain size decreases with the current density. He also provided a relation between the average growth velocity $v_{g}$, the grain size $R_{g}$ and the grain growth time $T$ : $v_{g}=2 R_{g} / T$. This equation enables access to the nucleation frequency $T^{-1}=v_{g} /\left(2 R_{g}\right)$. This law shows that $T^{-1}$ increases with the current density, the latter causing $v_{g}$ to increase and $R_{g}$ to decrease, Fig. 1 . However, this evolution of the nucleation frequency is not consistent with the formation of dendrites in the limit of high current densities. Indeed, the dendrites are formed/built only by growth without renucleation events implying that $T^{-1}$ must vanish at high current densities and consequently a transition from the re-nucleation/growth regime (ramified branches) to the growth regime (dendrites) as sketched in Fig. 1. In this paper, this intrinsic transition between the two regimes is studied experimentally (SEM observations of the branches) and theoretically at the scale of the branch microstructure.

The inner wall of the cells used in this study is not activated (as in the work of Fleury ${ }^{39}$ ); indeed, a set of preliminary experiments shows that both the pattern and the microstructure of the branches are affected by the surface state of the cell wall. A specific method, based on the freezing of the cell, is used here to recover the nonadherent branches without damage. Current densities higher than these used in Fleury's work, were applied to cover both regimes. The main part of the study concerns the growth of copper branches and some experiments are performed with silver, to investigate the effect of the nature of the deposited metal.

As for the theoretical part, the onset of dendritic growth is analyzed by considering the required shape instability of the growing material. This is done by adapting the Mullins \& Sekerka shape stability model ${ }^{42}$ to the electrochemical situation. The effect of the operating parameters (current density $j$ and metal salt concentration $c_{0}$ ) on the obtained instability threshold is discussed and compared to experiment results. 


\section{Experimental}

Chemicals.-The aqueous metal salt solutions ( 0.1 to $0.75 \mathrm{M})$ are made by dissolving either copper (II) sulfate pentahydrate ( $>98 \%$, Sigma Aldrich) or silver nitrate ( $>99 \%$, Acros Organics) in deionized water $(18.2 \mathrm{M} \Omega . \mathrm{cm})$. Prior using the solutions, the dissolved oxygen is removed by bubbling nitrogen ( 1 bar) for $10 \mathrm{~min}$. (volume of solution $\sim 5 \mathrm{ml}$ ). The deaerated solutions are then collected by a gastight syringe (Hamilton $1 \mathrm{ml}, 1001 \mathrm{LT}$ ) and injected into the Hele-Shaw cell.

Experimental set-up.-The experimental set-up (Fig. 2) is made from two microscope glass plates $(76 \times 56 \times 1 \mathrm{~mm})$ which sandwich the electrodes in the form of two thin sheets of metal. The depth of the Hele-Shaw cell therefore corresponds to the thickness $e$ of the electrodes $(50 \mu \mathrm{m})$. The length and width $L$ of the electrolytic compartment are respectively $75 \mathrm{~mm}$ and $15 \mathrm{~mm}$. The same metal element was used for both electrodes (metallic form) and for the electrolyte (dissolved metal salt). The purity of the metal electrodes is $99.9 \%$ for both copper and silver (Goodfellow). To avoid leakage between the electrodes and the glass plates, the inner sides of the glass plates are covered by a transparent laboratory parafilm. The effect of the functionalization of the inner cell walls is investigated by metallizing the parafilm by gold vapor deposition. The obtained gold coating is non-percolating (as in Fleury's work) as confirmed by resistivity measurements.

The electrodes are polished with polishing paper and cleaned with ethanol and then flushed with deionized water before each use. The two remaining opening sides of the cell are closed by applying a reusable adhesive paste (UHU patafix). Fluidic connections are made using nanoport connectors (Idex-hs) on holes drilled in the glass (Dremel diamond wick). The cell is kept horizontal in a holder and filled with the electrolyte solution previously collected with the syringe. After each experiment, the device is dismantled, cleaned and re-assembled for the next experiment.

The galvanostatic electrolyses are performed using either a potentiostat (Autolab PGSTAT100N) or a current generator (TDKLambda Gen2400W) to apply an electric current ranging from 1.25 to $20 \mathrm{~mA}$ ( $j$ is in the range 33 to $266 \mathrm{~mA} \mathrm{~cm}^{-2}$ ) between the electrodes, regardless of the electrical resistance of the system. All experiments are performed under ambient conditions $\left(\sim 20^{\circ} \mathrm{C}\right)$.
Branch recovery method.- Just after the electrochemical formation of the metallic branches, a cooling spray (RS components 846-682), inducing a temperature decrease of $\sim 50{ }^{\circ} \mathrm{C}$, is applied to the top glass plate of the device to freeze the liquid electrolyte. This step allows the inlet fluidic connection to be opened without disturbing the highly fragile electrodeposit, in order to connect a syringe of deionized and deaerated water. After, the cell is left to unfreeze and return to room temperature, and a flow is applied for 30 min to gently flush the metallic branches $\left(\sim 20 \mu 1 \mathrm{~min}^{-1}\right)$ using a syringe pump (Harvard Apparatus PhD Ultra 70-3006). This allows the removal of almost all the electrolyte thus avoiding any eventual crystallization in samples examined by SEM.

The cell is then frozen again, with the cooling spray, to disconnect the two fluidic connections before putting the entire system in a freezer for $\sim 30 \mathrm{~min}$. Next, the cell is opened quickly on an ice bed and the branch pattern is transferred to an adhesive carbon tape, where it is left free to dry under ambient conditions. This method allows the recovery of branches with little disturbance and their analysis by SEM on a wide range of length scales (from $\sim 1 \mathrm{~mm}$ up to $\sim 10 \mathrm{~nm}$ ), as shown in Fig. 3 .

Visualization of the branch pattern.-The growth of the branches is visualized by transmission using a LED panel, placed below the device, and a camera PCO pixelfly, associated with a 105 $\mathrm{mm}$ macro lens, facing the top glass plate. The acquisition frequency is 1 image $\mathrm{s}^{-1}$ unless another value is specified. The ImageJ software and python scripts are used for image processing.

Characterization of branch microstructure.-All the branch samples are metalized by vapor deposition of a layer of $\sim 10 \mathrm{~nm}$ of gold or platinum (duration $=60 \mathrm{~s}$, vacuum $=10^{-1} \mathrm{mbar}$ ) before being observed by SEM using a MEB-FEG JEOL JSM 7800F Prime -EDS or a JEOL JSM 7100F TTLS.

\section{Results and Discussion}

Several experiments are carried out, varying $j$ and $c_{0}$, and the resulting ramified branches are observed by both optical visualization and SEM. Additionally, this is done with and without metallization of the cell wall to investigate its effect on the branch microstructure. The corresponding results are first discussed in the

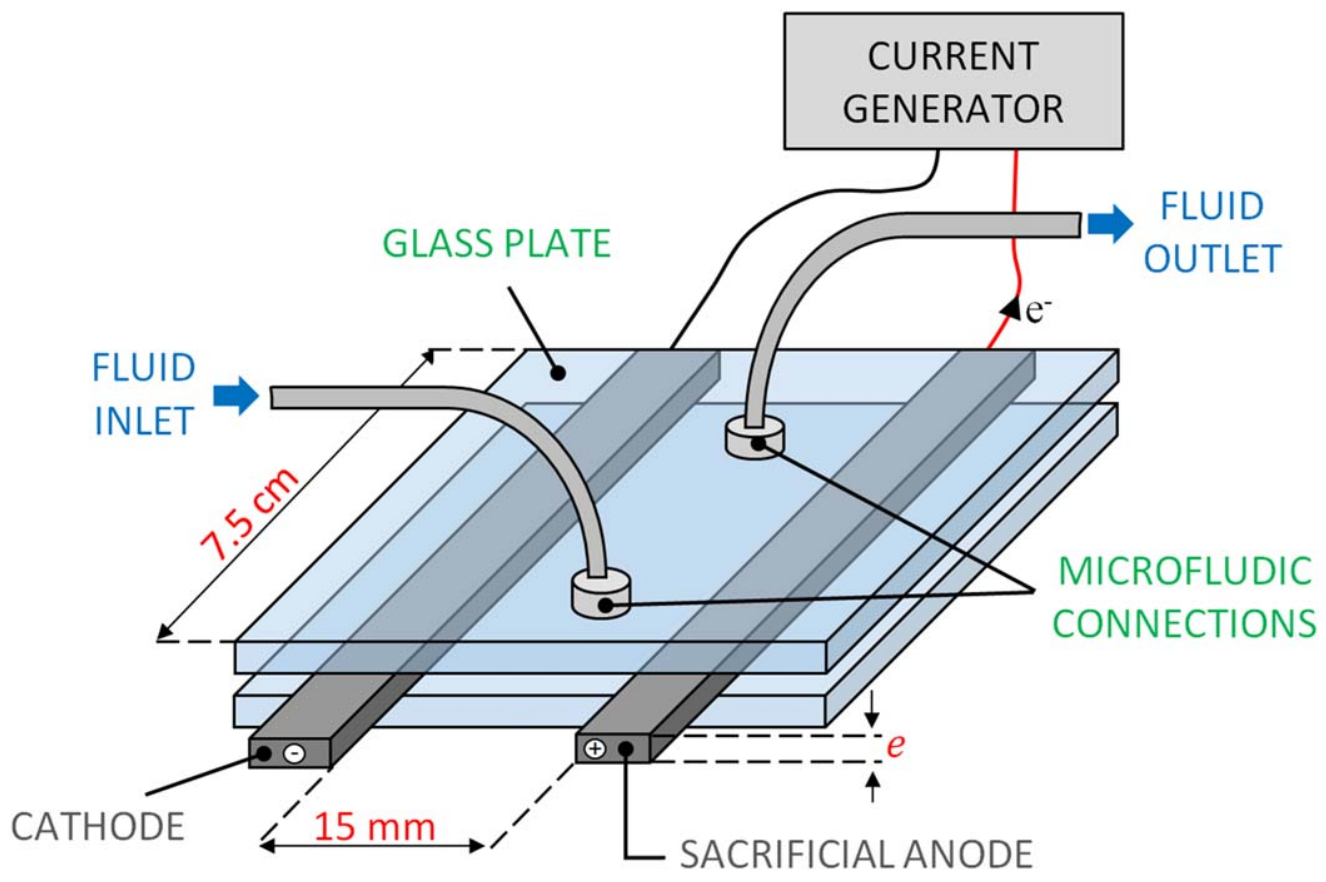

Figure 2. Sketch of the experimental set-up. 

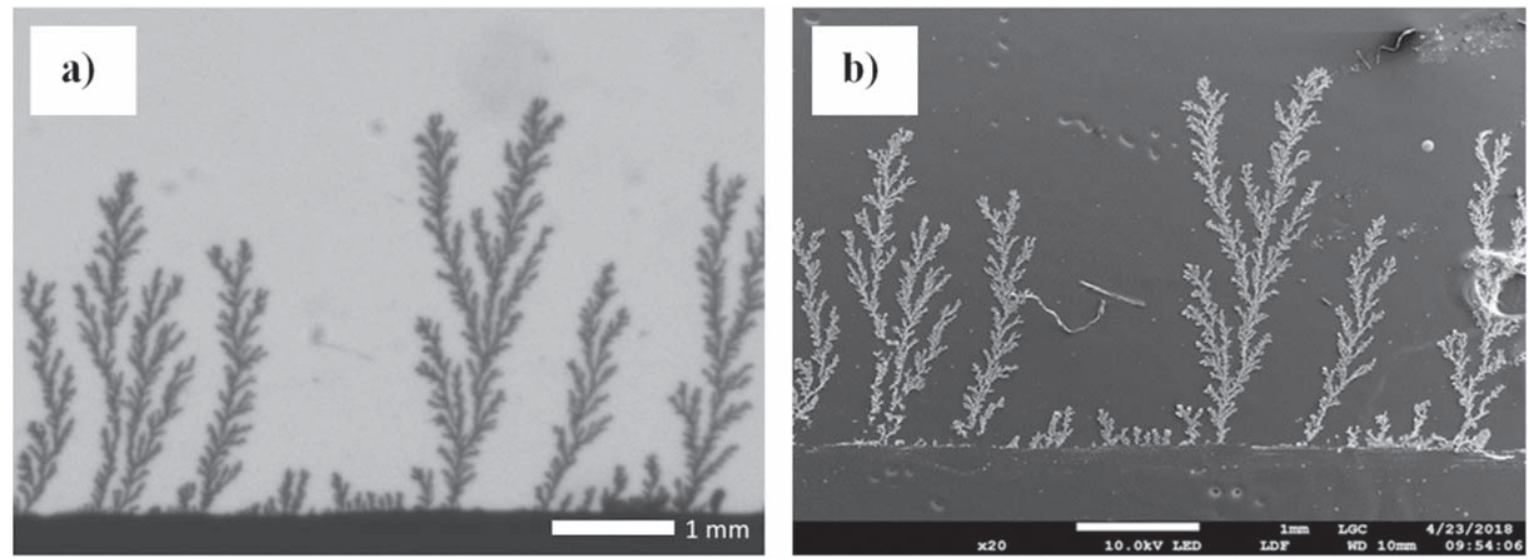

Figure 3. (a) Optical image of ramified copper branches taken during a galvanostatic electrolysis in the Hele-Shaw cell (elapsed time $=220 \mathrm{~s}, c_{0}=0.5 \mathrm{M}, j=$ $133 \mathrm{~mA} \mathrm{~cm} \mathrm{~cm}^{-2}$ and non-metallized cell wall), (b) SEM image taken after the recovery of the deposit.

following sub-section. Then, the results obtained without metallization are given and analyzed in the subsequent sub-sections.

Effect of cell wall metallization on branch microstructure.The microstructures of copper branches, obtained for $j=$ $133 \mathrm{~mA} \mathrm{~cm}^{-2}$ and $c_{0}=0.5 \mathrm{M}$, with and without cell wall metallization are shown in Fig. 4. With metallization, as already reported in previous works, ${ }^{43,44}$ the branches adhere to the cell wall; the deposit is thus easily recovered, flushed and analyzed by SEM (as in the Fleury's work ${ }^{39}$ ). The corresponding SEM images, Figs. 4a-4b, show that the branches have the form of "compact tongues" composed of small grains (equivalent diameters range from $\sim 100$ to $\sim 600 \mathrm{~nm}$ ). Without metallization, the branches do not adhere to the cell wall; their recovery and SEM analyses are achieved from the method described in the section Experiemental. In this case, the branch microstructure is different and exhibits an expanded and dendritic structure, Fig. 4c-4d. The same differences, with and without metallization, have been
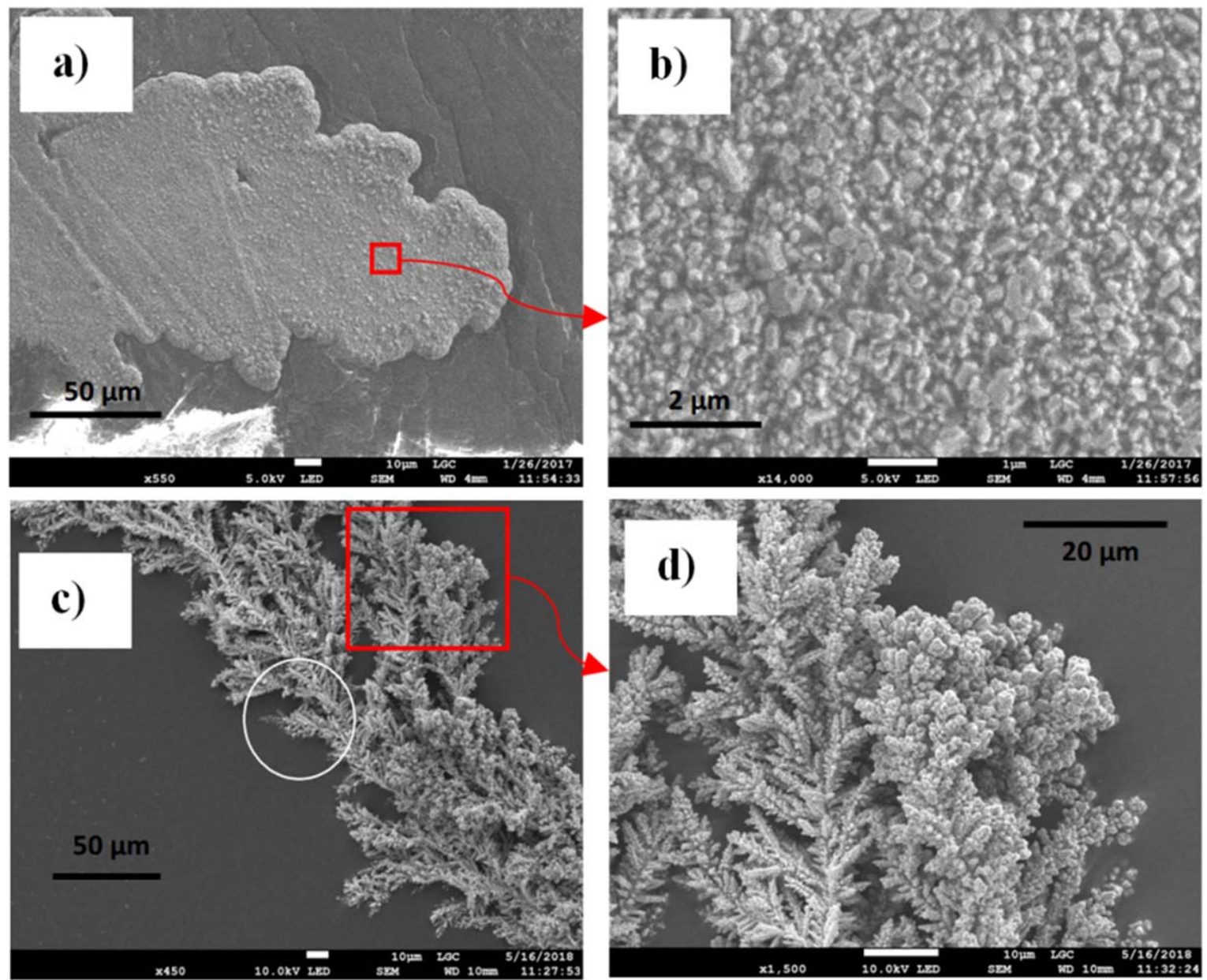

Figure 4. SEM images of copper branches obtained with gold metallization of cell walls, at a magnification of $\times 550$ in a) and $\times 14000$ in (b), and without metallization (with cell walls made of bare parafilm), at magnification of $\times 450$ in (c) and $\times 15000$ in (d); $j=133 \mathrm{~mA} \mathrm{~cm}-2$ and $c_{0}=0.5 \mathrm{M}$. 
observed when varying the values of the operating parameters $(j$ and $c_{0}$ ) in the investigated ranges.

These results show a strong influence of the interaction between the cell wall and the electrodeposited metal on the branch microstructure. In the case of adherent branches (with metallization), the growth phenomenon is complex since the microstructure should clearly depend on the properties of the metallized film (nature of the metal, size distribution, average thickness, etc); these properties must therefore be considered for a full description of the branch growth as initiated in previous works. ${ }^{43,44}$

This work focuses on the study of the still poorly explored intrinsic transition between ramified branches and dendritic deposits (at the scale of the microstructure) regardless of the surface state of the cell wall. Consequently, the study is performed with nonadherent branches obtained without metallization of the cell wall

Morphology of non-adherent branches at the macro-scale.Fractal property.-Figure 5 (left column) shows optical images of the obtained non-adherent ramified branches by electrolysis of a $0.5 \mathrm{M}$ copper sulfate solution at three values of $j, 66 \mathrm{~mA} \mathrm{~cm}^{-2}$, $133 \mathrm{~mA} \mathrm{~cm}^{-2}$ and $266 \mathrm{~mA} \mathrm{~cm}^{-2}$. As usually observed, these copper deposits are ramified patterns of DB type. The fractal property of these deposits is determined by using a two-dimensional box counting algorithm (ImageJ). The corresponding curves of the number of boxes (count), which covers the deposit, as a function of the box size are
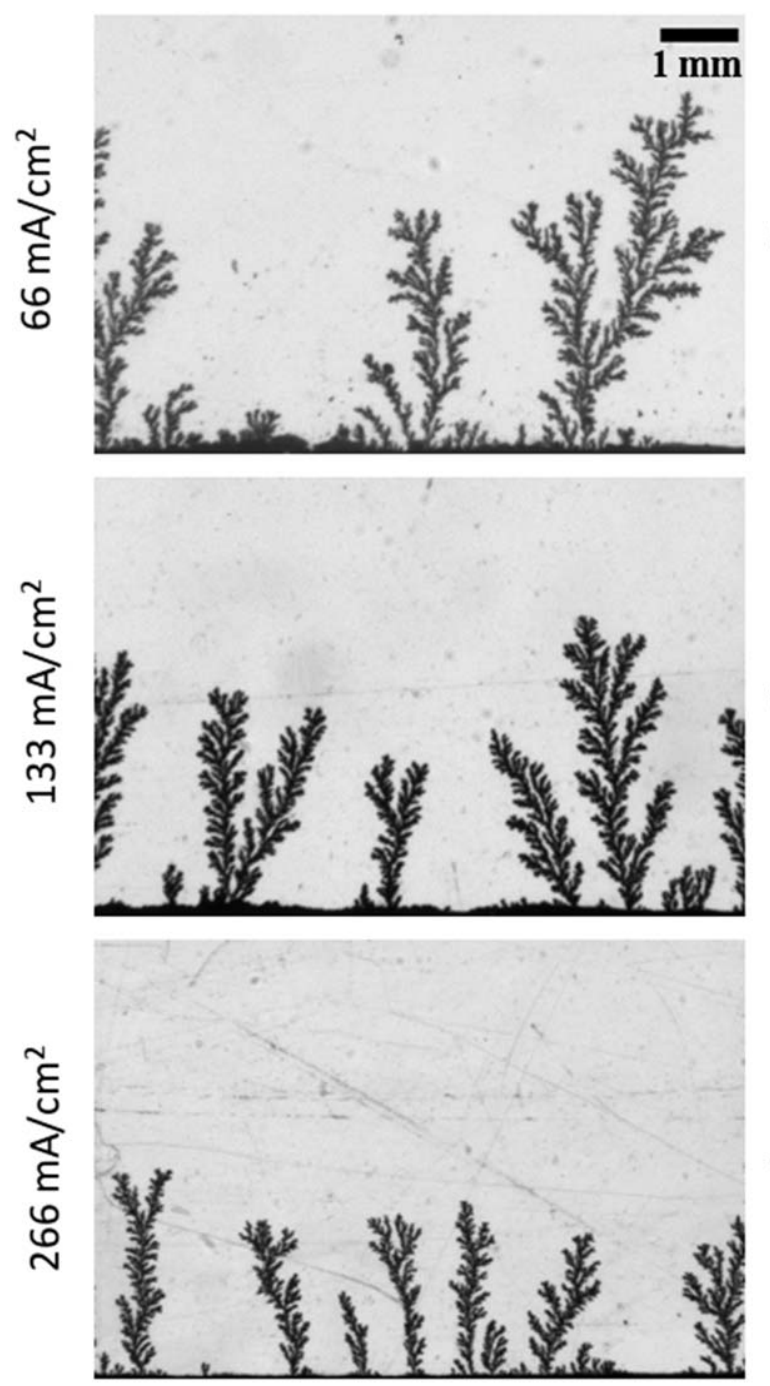

plotted, using a log-log scale, in the right column of Fig. 5. Concerning the range of the box size, the minimum value is set to 2 pixels $(\sim 20 \mu \mathrm{m})$ and the maximum value used, of 256 pixels ( $\sim 2 \mathrm{~mm}$ ), is selected in such a way that the count remains higher than 10 (to keep a representative sampling). The linear curves obtained, and their slope $>-2$, show that the deposits are fractal in the corresponding range of length scales $[\sim 20 \mu \mathrm{m}, \sim 2 \mathrm{~mm}]$, i.e. at the macro-scale, as expected. A same value of the fractal dimension $D_{f}$ (given by the slope $d \log ($ count $) / d \log \left(\right.$ box $\left.\operatorname{size}^{-1}\right)$ ) of $\sim 1.61$ is obtained for each case (Fig. 5, right column). This value of $D_{f}$ corresponds to the values typically reported for both ramified electrodeposits ${ }^{26}$ and DLA patterns ${ }^{29}$ and this shows that, as expected, branch growth is mainly limited by diffusion, at the macro-scale.

Average growth velocity and deposit density.-The average growth velocity $v_{g}$ of these DB patterns is expected to correspond to the anion velocity: ${ }^{13}$

$$
v_{g}=-z \mathrm{~F} u_{a} E,
$$

where $z$ is the ion valence, $\mathrm{F}$ the Faraday's constant, $u_{a}$ the mobility of anions and $E$ is the electric field in the bulk electrolyte (away from the electrodes). In this region, the electrolyte has not yet been consumed to make grow the deposit and thus the concentration field is uniform (this is justified because the applied current is higher than
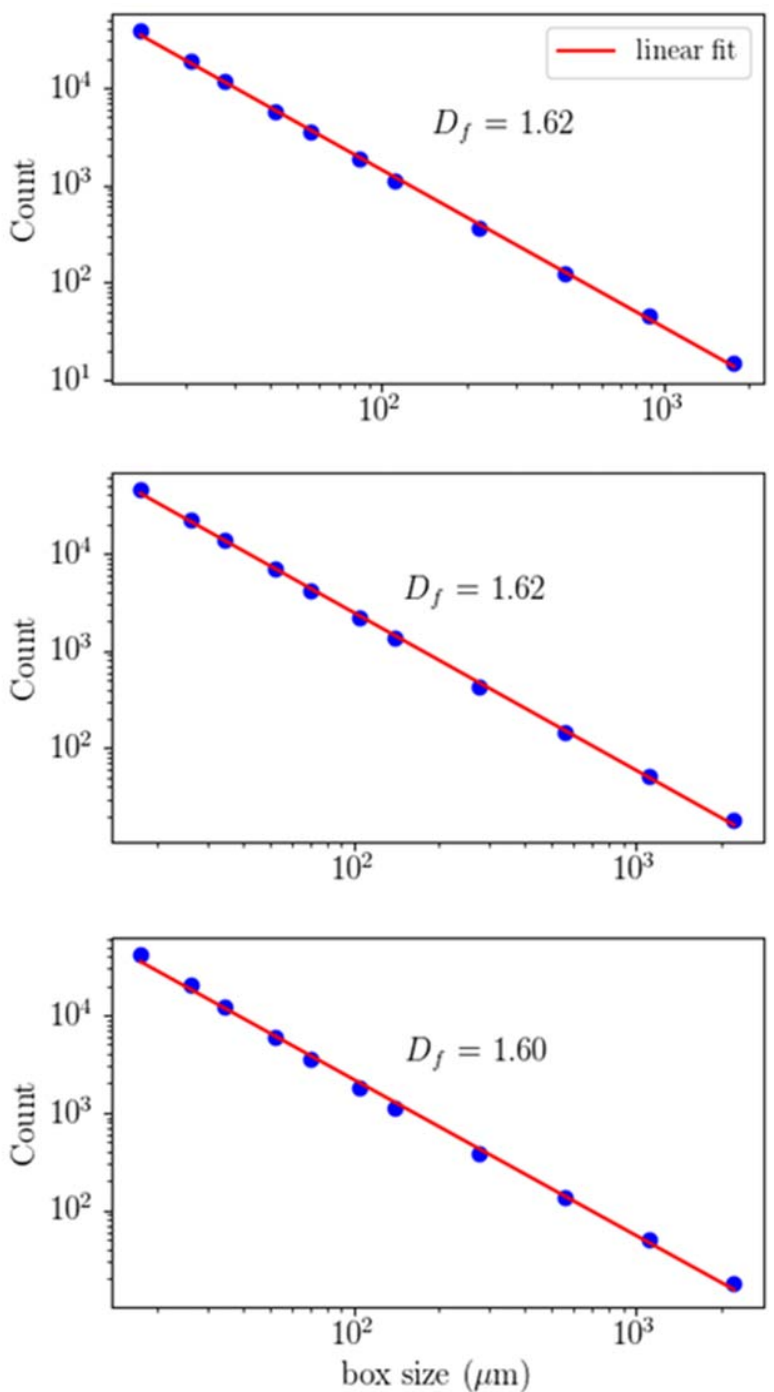

Figure 5. Left: optical images of ramified copper deposits obtained with $c_{0}=0.5 \mathrm{M}$ and several $j$ values. Right: the corresponding plots of the box counting method (ImageJ) to highlight the fractal property and to measure the fractal dimension $D_{f}$ (indicated inside each graph). 
the limiting current which is based on the concentration gradient given by the ratio between $c_{0}$ and the distance between the initial electrodes $\left.{ }^{12}\right)$. As a consequence, in this region, Ohm's law applies, $j=-z^{2} \mathrm{~F}^{2}\left(u_{a}+u_{c}\right) c_{0} E$ (where $u_{c}$ is the mobility of cations), and Eq. 1 can be given in a form in which the operating parameters, $j$ and $c_{0}$, appear explicitly: ${ }^{15}$

$$
v_{g}=\frac{1-t_{c}}{z \mathrm{~F}} \frac{j}{c_{0}},
$$

where $t_{c}$ is the transference number of cations.

$v_{g}$ is experimentally measured from the temporal evolution of the average front location $\bar{x}_{f}(t), v_{g}=d \bar{x}_{f} / d t . \bar{x}_{f}(t)$ is determined from the density profiles $\rho_{d}$ which are computed from the optical images as $\rho_{d}(x)=\int_{0}^{W} A(x, y) d y / W$, where $W$ is the width of the image, $A=1$ where there is metal and $A=0$ elsewhere ( $x$ the coordinate along the axis perpendicular to the initial surface of the cathode and directed towards the anode, and $y$ the coordinate along the axis parallel to the initial electrode surfaces). In Fig. 6b, the density profiles $\rho_{d}$ are plotted, at several growth times, for a copper deposit obtained with $c_{0}=0.5 \mathrm{M}$ and $j=266 \mathrm{~mA} \mathrm{~cm}^{-2}$; the corresponding final image of the deposit is shown in Fig. 6a. The branch growth quickly reaches a steady state characterized by the S-shaped curve of the density profiles which is uniformly translated along the $x$ axis (Fig. 6b). The average front location $\bar{x}_{f}$ is then defined and measured from the position of the inflection point of these curves, as sketches in Fig. 6b for the last profile (this position is also shown in the optical image of Fig. 6a). As already reported, ${ }^{15,21} \bar{x}_{f}$ evolves linearly with time after the initial depletion phase ${ }^{15,45}$ (Sand's time), see the insert in Fig. $6 b$.

Note that all the S-shaped density profiles have the same amplitude (Fig. 6b), which means that the growth front moves by leaving behind a deposit of constant density. This indicates that the deposit is no longer fractal above a given length scale. This is characteristic of DB patterns and is explained by the scaling effect of the growth front by the diffusion length which decreases when $j$ (or the mass flux) is increased. ${ }^{13,15,30-32,46}$ The length scale above which the deposit is non-fractal can be estimated to be $\sim 1 \mathrm{~mm}$, from the width of the wave of the $\rho_{d}$ profiles for $j=266 \mathrm{~mA} \mathrm{~cm}^{-2}$, Fig. $6 b^{30,32,46}$ For lower $j$ values, this length scale is even higher (this is indeed visible in the optical images of Fig. 5, where the branch density decreases when $j$ decreases). This explains why the non-fractal property is not captured by the box counting method (Fig. 5, right column) in which the larger length scale does not exceeds $2 \mathrm{~mm}$ whereas at least an additionally order of magnitude would be necessary to capture it.

In Fig. 7, the measured values of $v_{g}$ are plotted as a function of the ratio $j / c_{0}$ for all the investigated cases $(0.10,0.25,0.50$ and $0.75 \mathrm{M}$ ) for $c_{0}$ and $\left.j \in\left[33,266 \mathrm{~mA} \mathrm{~cm}^{-2}\right]\right)$. As expected, the points align along a linear curve, in accordance with Eq. 2. From a linear fit, we obtained for $t_{c}$ a value of 0.393 , that is also in accordance with other works on copper branches. ${ }^{15}$ Note that $v_{g}$ corresponds to the highest local growth velocity on the contour of the branches; the branch tops grow fastest at $v_{g}$ (Fig. 6a).

In the next section, the microstructure of these ramified branches is observed and analyzed by SEM.

Microstructure of non-adherent branches.-Effect of the current density.-Figures 8 and 9 show the SEM images of the microstructure of the obtained ramified branches by electrolysis of a $0.5 \mathrm{M}$ copper sulfate solution at two values of $j, 33 \mathrm{~mA} \mathrm{~cm}^{-2}$ and $266 \mathrm{~mA} \mathrm{~cm}^{-2}$. Multiple magnifications of a same region are used to properly show the microstructure up to the lower cut-off length scale.
For $j=33 \mathrm{~mA} \mathrm{~cm}{ }^{-2}$ (Fig. 8), at low magnification $(\times 45$ and $\times 370$ ), the branch appears to be spongy/powdery. At $\times 370$ magnification, some regions (blue square, in the right column) exhibit a dendritic structure more easily seen by getting closer at $\times 2,500$ (black dashed line ellipses, in both columns). The images obtained at $\times 10,000$ magnification show that these dendritic structures consist of grain assemblies and that the grains are the smallest particulate constituents of the branches. These grain assemblies appear to be randomly ordered, but some of them show an apparent ordered arrangement and appear exhibiting a dendritic shape (black dashed line ellipses). Indeed, because these grain assemblies are composed of several grains, i.e. single crystals, they are not dendrites (a dendrite is a single crystal), and their mode of formation is different from that of a dendrite. Besides, the images obtained with $\times 2,500$ and $\times 10,000$ magnifications, show the presence of only a few real single dendrites (see black rectangles and inserts); these real dendrites are recognizable by their characteristic sharp apex and the symmetry of their geometry. Let's go back to the main morphologies observed. The grain assemblies that exhibit an ordered arrangement, so called dendritic structures here, arise from a combination of both re-nucleation and crystal (dendritic) growth. It can be concluded that these dendritic structures result from the growth of dendrite arms, on which re-nucleation events occurs continuously. Consequently, it seems difficult to differentiate between random grain assemblies and grain assemblies exhibiting a dendritic shape (dendritic structures). The re-nucleation process probably overcomes dendrite growth, for this current density. However, the dendritic structures cannot be neglected, as they could be well visible in some regions of the branch. In the next paragraph, a different microstructure is observed at high $j$.

The obtained branches at higher current densities exhibit a different microstructure as shown in Fig. 9 for $j=266 \mathrm{~mA} \mathrm{~cm}^{-2}$. Dendritic structures are now clearly observed, more frequently, and at lower magnifications. They are found in the central part of the main branch (see for example, the image at a magnification of $\times 5,000$, right column). Additionally, grain assemblies are also well visible but in localized regions corresponding to the ends of sub-branches (see the image at a magnification of $\times 5,000$, left column). However, even if dendritic structures appear more clearly, and look like single dendrites, they are not single dendrites. They cannot be considered as single crystals because they do not exhibit the characteristic symmetry of dendrites (already observed with single dendrites for $33 \mathrm{~mA} \mathrm{~cm}^{-2}$, black rectangles and inserts in Fig. 8 at $\times 2,500$ ). A mixed growth, combining re-nucleation and dendrite growth, also applies for this current density. Remind that in the absence of re-nucleation a single (dendritic) crystal must be observed; here the deposit appears as a forest of dendritic structures of which the growth direction can change suddenly as indicated by the gray lines at $\times 5,000$ in Fig. 9 . This can only be explained by still ongoing re-nucleation events which allow the starting of new dendritic structures and freedom in growth direction. The continuous re-nucleation, during the growth of a dendrite, is clearly shown in Fig. 10, where freshly nucleated crystals are observed on the top of a dendritic structure (for an intermediate current density of 133 $\mathrm{mA} \mathrm{cm} \mathrm{cm}^{-2}$ and $\left.0.5 \mathrm{M}\right)$. The resulting random growth direction is compatible, and even required, with the fractal property of the pattern at the macro-scale (Fig. 5). At the microstructure scale, if only dendrites would be formed (without re-nucleation process), the pattern cannot have the same fractal and isotropic properties because of the too limited growth directions allowed by the purely anisotropic crystal growth. The re-nucleation process allows the connection between the isotropic and fractal structure, at the macroscale, and the anisotropic structure, at the microscale.

To have a better view of the spatial distribution of the two main particulate morphologies (grain assemblies and dendritic structures) all along a branch, a region of a branch is first scanned with an intermediate magnification $\times 370$, Fig. 11 . Next, the resulting images 

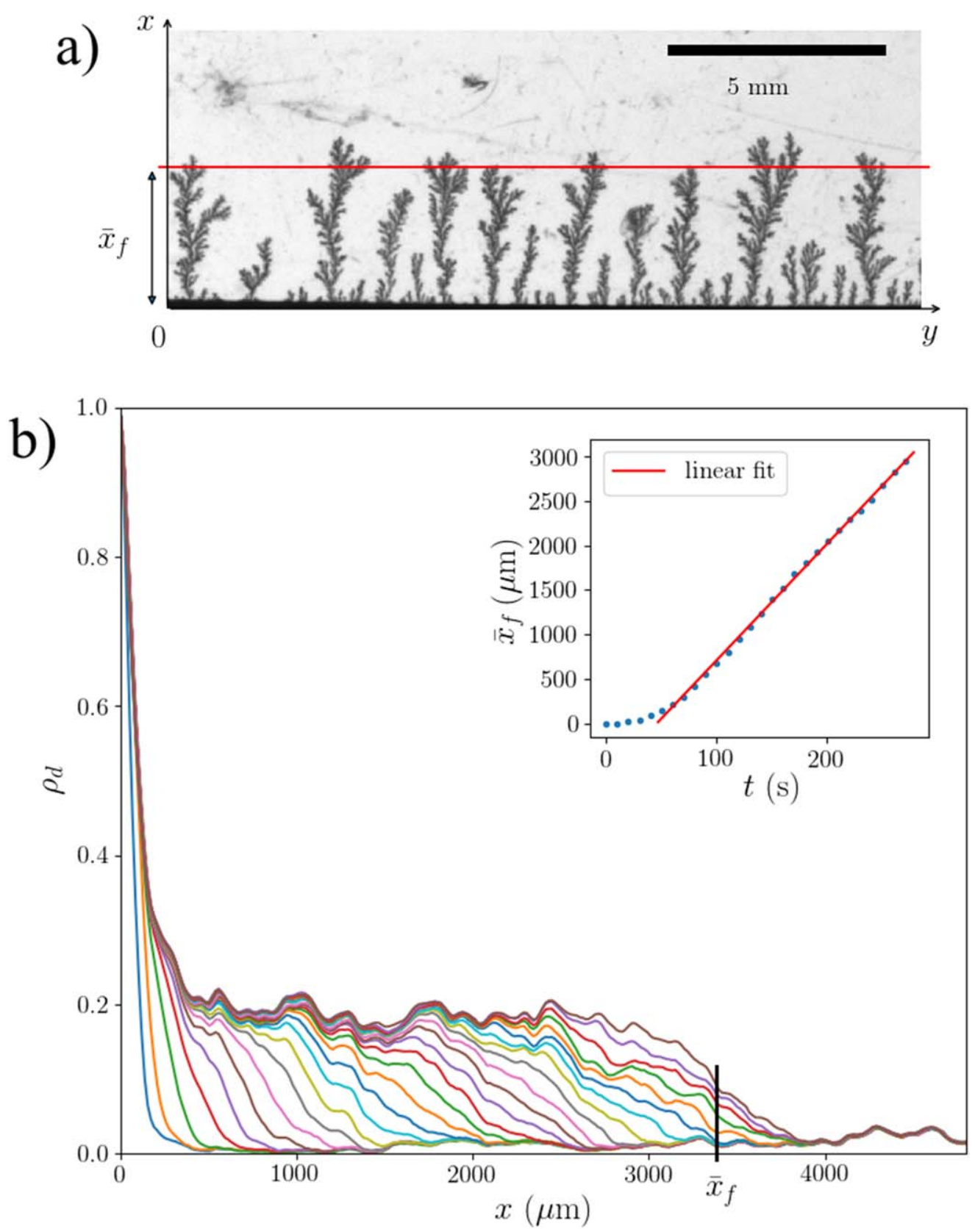

Figure 6. (a) Optical image of a ramified copper deposit obtained with $c_{0}=0.5 \mathrm{M}, j=266 \mathrm{~mA} \mathrm{~cm} \mathrm{~cm}^{-2}$ after an electrolysis duration of $280 \mathrm{~s}$, the red line indicates the average front location $\bar{x}_{f}$ detected from the inflection point of the last S-shaped density profile shown in (b). (b) The corresponding density profiles $\rho_{d}$ at several times (10 s between each curve), the insert shows the temporal evolution of $\bar{x}_{f}$.

are processed (automatic thresholding, particle detection and morphology properties) to generate an image in which each particle region is colored as a function of a morphology property. Here, the solidity is chosen to differentiate between grain assemblies and dendritic structures. The solidity of a particle corresponds to the ratio between the projected area of the particle divided by the area of the corresponding hull image (smallest convex polygon enclosing the particle). For a grain, the shape is rounded and so the solidity is close to 1 . On the contrary, for a dendritic structure, the solidity is markedly smaller than 1 .

By comparing the solidity maps, for 33 and $266 \mathrm{~mA} \mathrm{~cm}^{-2}$ (Fig. 11), it is highlighted that:

- at high $j$ (266 mA cm ${ }^{-2}$, Fig. 11b), dendritic structures (purple and blue particles) are located in the core of the branch while, as already noticed above, grain assemblies (yellow and white particles) are found mainly at the ends of sub-branches; the branch has a broccoli structure

- at low $j$ (33 mA cm ${ }^{-2}$, Fig. 11a), both particulate morphologies are uniformly distributed.

The two growth modes, the growth by re-nucleation and the dendritic growth, take place simultaneously, whatever $j$ values. It is observed that the re-nucleation growth mode is favored at low $j$ while the dendritic growth mode is favored at high $j$. Furthermore, this competition seems to apply locally. Indeed, grain assemblies are observed in ends of sub-branches which correspond to branch regions experiencing low local current densities, especially when they stop growing while the main branch continues to grow. Conversely, the central part of a branch has been formed faster by being subjected to higher local current densities. The latter regions are composed of dendritic structures, at high $j$. When $j$ is increased, 


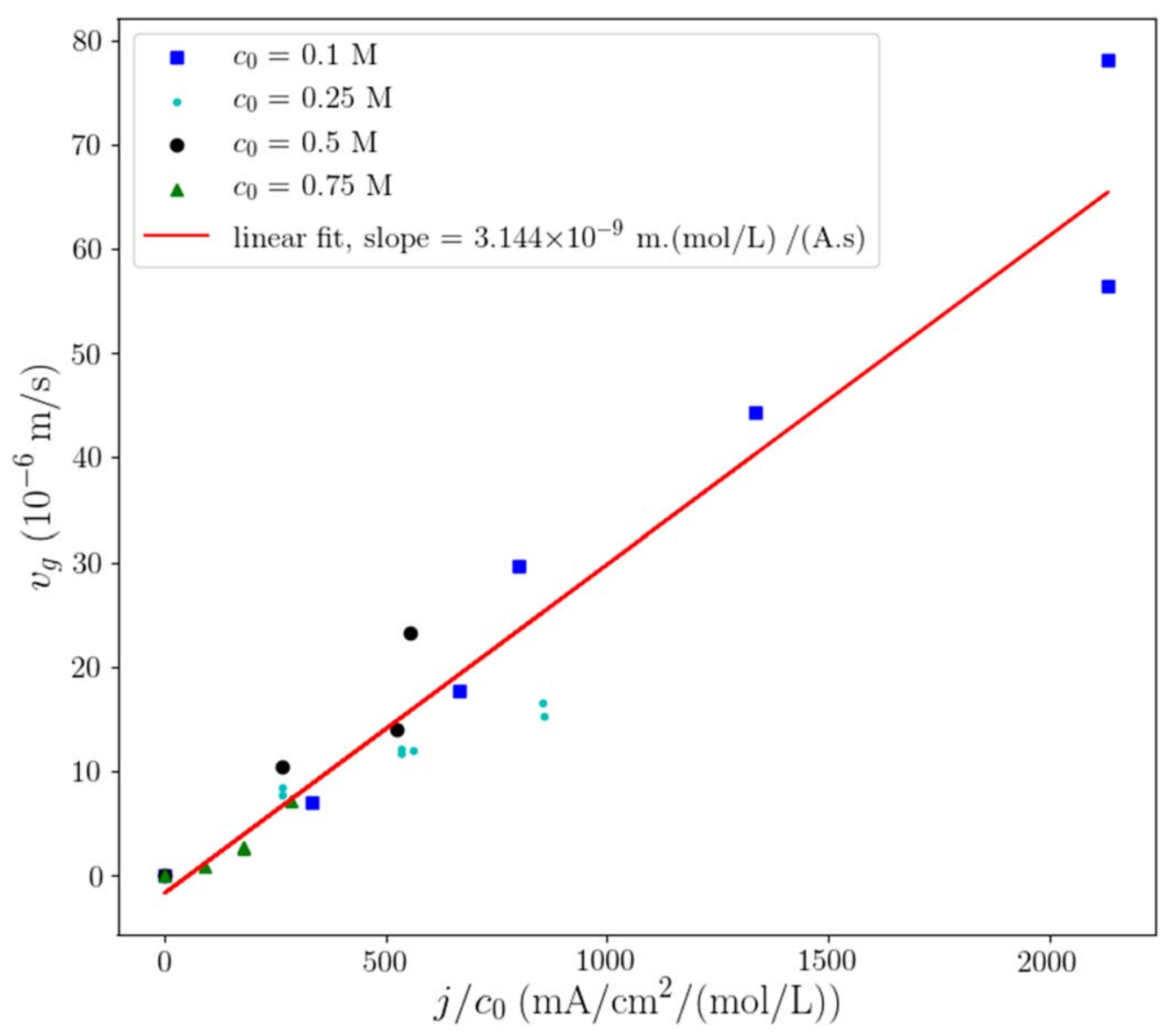

Figure 7. The average growth velocity $v_{g}$ as a function of the ratio $j / c_{0}$ for all of the investigated concentrations; the red line corresponds to the linear fit of all the points (the slope $\left.=3.144 \times 10^{-9} \mathrm{~m} .\left(\mathrm{mol} \mathrm{l}^{-1}\right)(\mathrm{A} . \mathrm{s})^{-1}\right)$.

the range of the local current density widens-from 0 to the average growth velocity $v_{g}$ (Eq. 2) which corresponds to the local growth velocity at the top of the branches (Figs. $6 \mathrm{a}$ and $6 \mathrm{~b}$ ) — and this results in an apparent spatial segregation of the two particulate morphologies (as observed in Fig. 11b). While, when $j$ is decreased, the current density range shortens ( $v_{g}$ decreases), that limits the appearance of dendritic structures everywhere. In the limit of low $j$, the re-nucleation growth mode dominates and branches are mainly composed of grain assemblies (note that the few single dendrites observed if Fig. 8 can be attributed to early but limited growth of dendritic structures). This corresponds to the growth mode described by Fleury. Note that, the Fleury's prediction concerning the increase of grain size with the decrease of the growth velocity, is observed locally at the ends of sub-branches: the grain size increases when going towards the end of a sub-branch, as indicated in Fig. 9 at $\times 5,000$.

Note that other branch patterns, obtained with intermediate $j$ values, were also observed by SEM. The obtained images are consistent with this analysis based on the two extreme cases (33 and $266 \mathrm{~mA} \mathrm{~cm}^{-2}$ ) by showing a progressive effect.

Effect of the concentration.-The effect of the concentration was also studied. This is shown in Fig. 12 where branches, obtained with $j=66 \mathrm{~mA} \mathrm{~cm}^{-2}$ and for two concentrations 0.25 and $0.75 \mathrm{M}$, are observed by SEM at several magnifications. We mainly observe dendritic structures for $0.25 \mathrm{M}$ and grain assemblies for $0.75 \mathrm{M}$. It is interesting to note that, dendritic structures are observed even at the ends of sub-branches contrary to the previous case $0.5 \mathrm{M}$ and $266 \mathrm{~mA} \mathrm{~cm}^{-2}$. This is observed while the average growth velocity $v_{g}$, which is proportional to the ratio $j / c_{0}$ (Eq. 2) and corresponds to the maximum local growth velocity around a branch, is lower for
$0.25 \mathrm{M}$ and $66 \mathrm{~mA} \mathrm{~cm}^{-2}$ than for $0.5 \mathrm{M}$ and $266 \mathrm{~mA} \mathrm{~cm}^{-2}$. This shows that the local microstructure does not only depend on the local growth velocity but also on the concentration $c_{0}$. When $c_{0}$ is decreased, the dendritic growth mode is favored over the renucleation process.

Effect of the nature of the metal.-Some experiments were carried out with silver instead of copper. Fig. 13 shows the microstructures of silver branches obtained for four values of $j$ $\left(33,66,133\right.$ and $\left.266 \mathrm{~mA} \mathrm{~cm}{ }^{-2}\right)$ and an electrolyte concentration of $0.25 \mathrm{M}$. At the lowest $j\left(33 \mathrm{~mA} \mathrm{~cm}^{-2}\right)$, branches appear composed of grain assemblies as for copper at low $j$ (re-nucleation growth mode dominates). For the other values of $j\left(66-266 \mathrm{~mA} \mathrm{~cm}^{-2}\right)$, the deposits consist of larger and elongated single crystals that shows that the dendritic growth mode predominates at these $j$ values; these elongated crystals are assimilated to dendrites since their shape differs from the equilibrium shape which would have an aspect ratio closer to 1 . The fact that the amount of re-nucleation events (apparently lower for silver) and the shape of the dendrites differ between copper and silver is probably related to a difference in anisotropy between these two materials. This shows that the properties of the deposited material affect the competition between both growth modes.

To sum up, for copper and silver, at low $j$, the branches consist of grain assemblies. Such a microstructure is attributed to the renucleation process described by Fleury. ${ }^{39}$ Increasing $j$ leads to the formation of dendritic structures at the scale of branch microstructure while at the macro-scale, the deposits exhibit a DB morphology by being isotropic and fractal. These dendritic structures arise from the combination of the two growth modes, re-nucleation process and dendritic growth. Decreasing the concentration leads to an earlier 


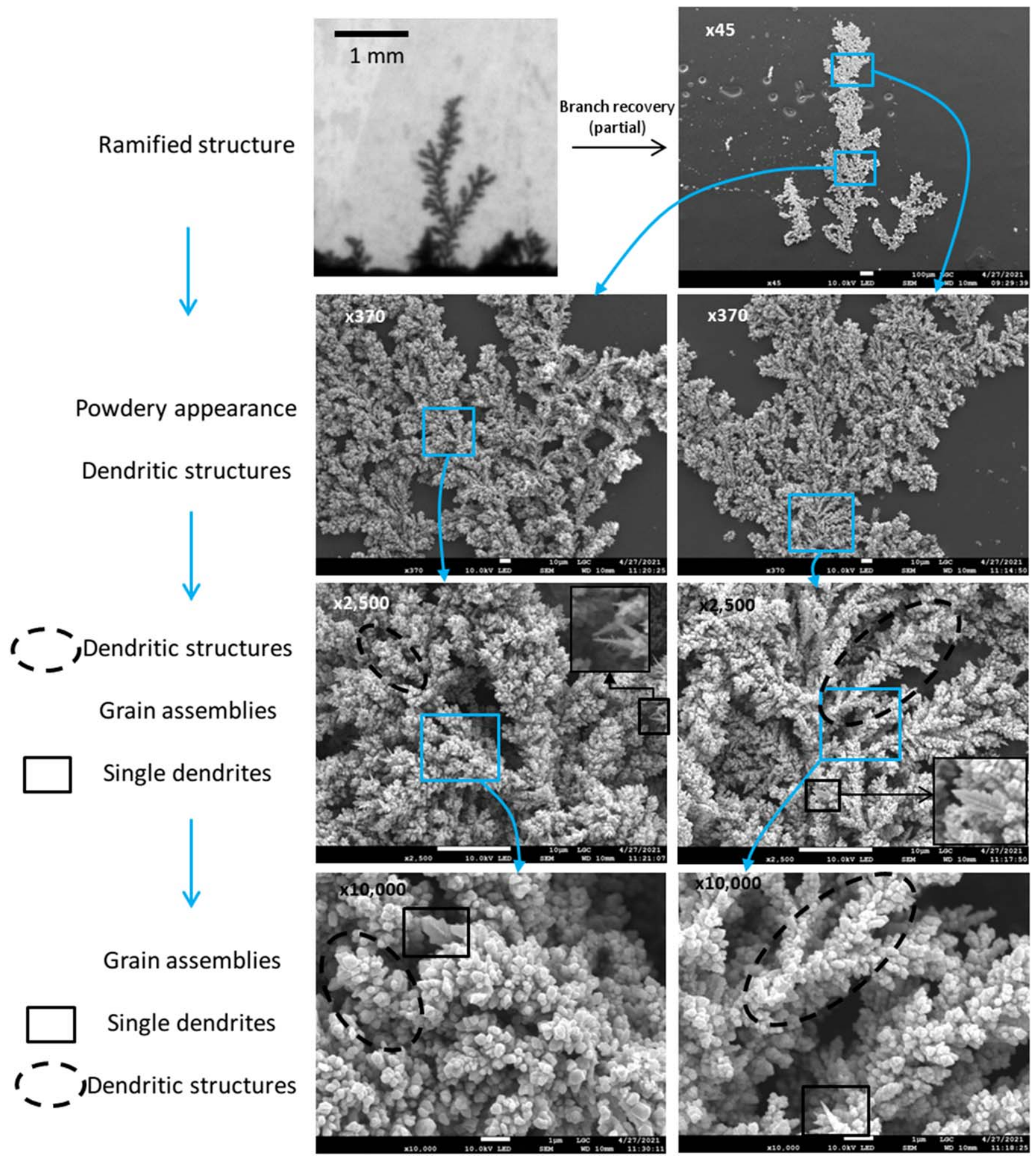

Figure 8. SEM images of a copper branch obtained for an applied current density of $33 \mathrm{~mA} \mathrm{~cm}{ }^{-2}$ and $c_{0}=0.5 \mathrm{M}$. Two regions are visualized by successively increasing the magnification. For each magnification, on the left, the main particulate morphologies observed are given in order of importance. In order to focus on the main result, only one kind of morphology is indicated per image by either black dashed line ellipses, for dendritic structures, or black rectangles, for single dendrites.

predominance of the dendritic growth mode over the re-nucleation process. The next section focuses on the origin of the dendritic growth mode at the scale of branch microstructure.

On the onset of the dendritic growth mode at the scale of the microstructure.-Origin of dendritic growth: shape instability.Why the dendritic growth mode proceeds inside the branches, when $j$ is increased and $c_{0}$ is decreased, is theoretically considered here. It is widely accepted that the onset of dendritic growth is fundamentally due to a shape instability during the growth of an initially stable shape growing material (whatever the initial shape), as described in several Refs. 47-49. The shape instability occurs when the amplification rate of infinitesimal protrusions is higher than their damping rate. The Mullins \& Sekerka model, ${ }^{42}$ adapted to diffusion-limited 


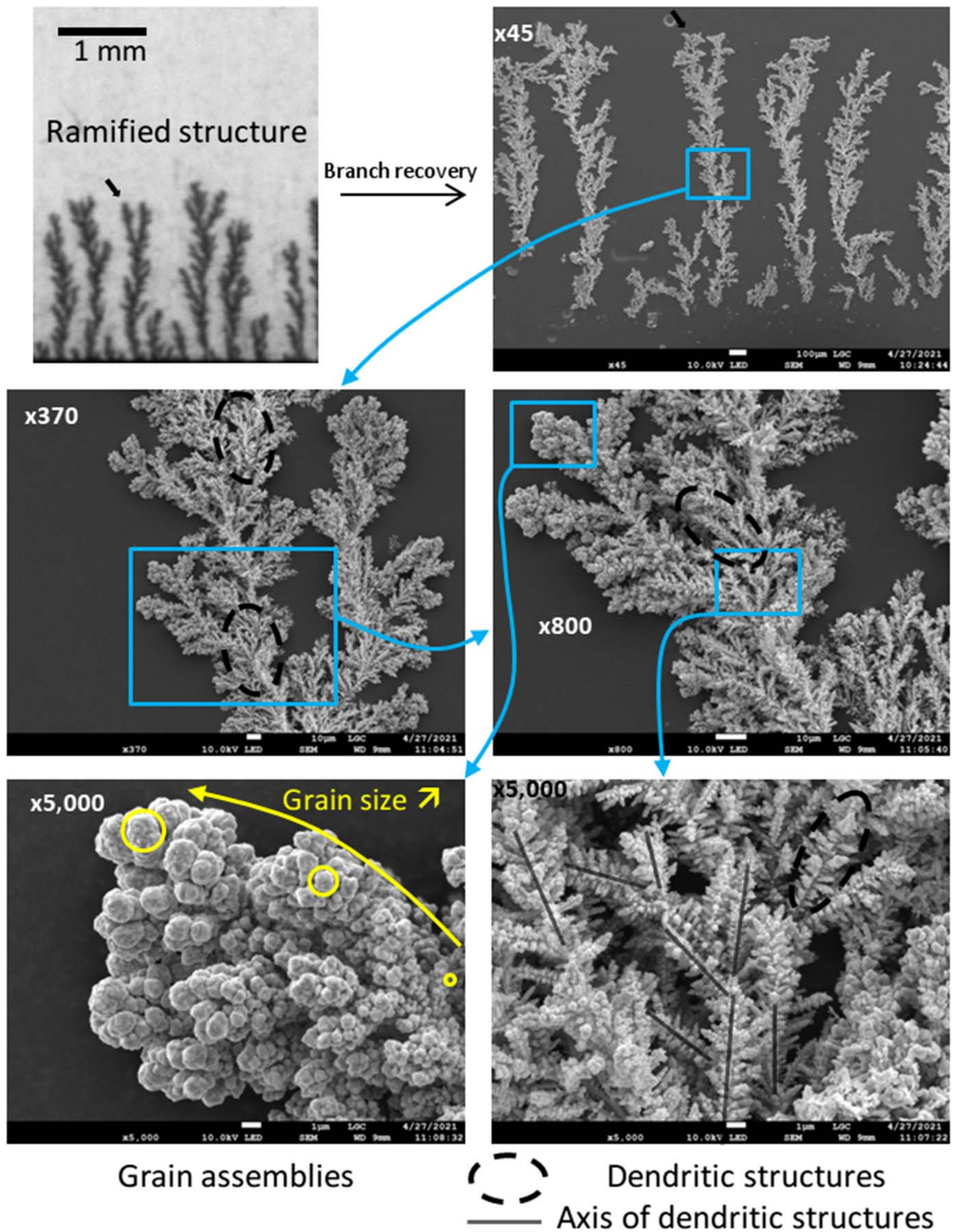

Figure 9. SEM images of a copper branch obtained for an applied current density of $266 \mathrm{~mA} \mathrm{~cm}^{-2}$ and $c_{0}=0.5 \mathrm{M}$. One region is visualized by successively increasing the magnification. In order to focus on the main result, only some dendritic structures are indicated per image by black dashed line ellipses. The growth directions of the dendritic structures are indicated by gray lines. 


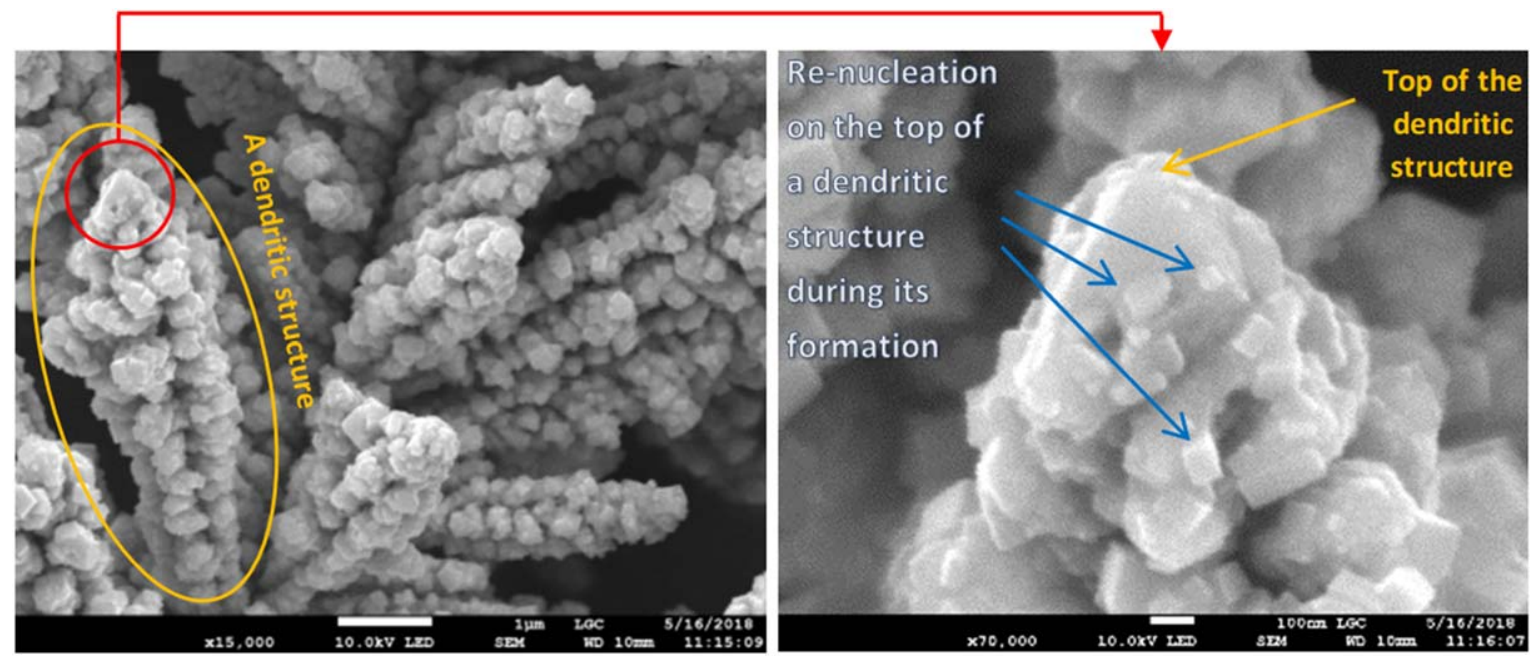

Figure 10. SEM images of a copper branch (central part) obtained with $0.5 \mathrm{M}$ and $133 \mathrm{~mA} \mathrm{~cm}{ }^{-2}$. This shows that the re-nucleation process is still present during the formation of copper dendritic structures.

growth, isotropic materials and fast kinetics, usually describes this mechanism. The amplification or the damping of shape fluctuations is dictated by the balance between destabilizing effects, generally a "point effect of diffusion", and stabilizing effects which are surface energy and kinetics. If the growth is slow, the stabilizing effects have time to damp any eventual deviations/protrusions to the stable shape and consequently the particle is stable keeping its equilibrium shape (non-dendritic particle). While if the growth is fast, the amplification rates of the protrusions are higher than the damping rates and the particle is unstable; this results in the formation of a dendrite.

The stability problem is formulated with a threshold which has to be exceeded for inducing dendritic growth. In the experiments this threshold is exceeded above a given $j$, from when some grains become unstable and start to grow as dendrites. By being combined with the still acting re-nucleation process, this leads to the formation of the dendritic structures.

Consequently, the shape stability of a growing grain (particle), idealized as spherical, is considered. We consider the shape stability threshold formulated in term of a threshold grain size $R_{t}$. If the size of the growing grain $R(t)$ stays lower than $R_{t}$, during its growth duration $T$, no dendritic growth can occur. Dendritic growth is considered possible if $R(T)=R_{g}>R_{t}$.

Concerning the spherical shape considered in this modeling, this is an idealization but this is not expected to affect the roles of the main physical effects. Indeed, as it could be seen on the SEM images of the microstructure, the (non-dendritic) grains are not spherical. Therefore, there is an anisotropic effect as expected for the deposited materials (also shown by the dendrites themselves). But, as discussed jut before, the onset of dendritic growth, even for anisotropic material, is also fundamentally due to a shape instability. ${ }^{48,50}$ This latter is usually described by a Mullins \& Sekerka like model in which orientation-dependent surface energy and kinetic coefficient are taken into account. In this case, the situation is more complex since the threshold depends on the orientation. ${ }^{50}$ In the present work, it is not relevant and necessary to take into account all the complexity brought by anisotropies. Because, even if this would be done, the derived threshold would show the same trend (as a function of the operating parameters) as if an isotropic case would be considered. This is due to the fact that the competition between the stabilizing and destabilizing effects remains intrinsically the same regardless of the taking into account of the anisotropy and therefore of the shape of the growing grain. Instead, an isotropic situation is considered, and so a spherical shape is considered, but the transport of cations around the growing grain is specifically considered.

In the following, from the Mullins \& Sekerka shape stability analysis, the developed modeling aims to derive an equation for $R_{t}$. The modeling of the growth and shape of the resulting dendrites (that would require the taking into account of material anisotropies) is not attempted. As it stands, the Mullins \& Sekerka shape stability analysis (adapted to crystallization, condensation, etc.) is not directly adapted to the electrochemical situation. Firstly, because species transport involves at least two fields (electrolyte concentration and electric potential) instead of just one. Secondly, because the driving force is the overpotential instead of the oversaturation. Electrochemical shape stability analyses have already been performed, notably in Refs. 51, 52, but they cannot be applied here because they are not adapted to the shape stability of the growing spherical grain: the initial shape is spherical and dynamic instead of being flat and the driving force (applied current or potential) varies during the growth instead of being constant. Consequently, a specific shape stability analysis is derived here.

Derivation of the threshold grain size from the adaptation of the model of Mullins \& Sekerka model to the electrochemical situation.-A growing grain at the top of a branch is assimilated to an initially spherical metal particle which grows, under the action of a cathodic polarization, in a stagnant solution of the metal salt (electrolyte), Fig. 14.

To enable the derivation of the stability analysis (using spherical harmonics, see below), the grain is considered as a single particle (note that the same simplification is used in the Fleury's model). The relevant scalar fields (concentrations and electric potential) are thus subject to spherical symmetry, Fig. 14. Eventual departure from the electroneutrality is not considered in this application of the Mullins \& Sekerka model. Consequently, the cation concentration $c^{+}$is equal to the anion concentration $c^{-}$in the solution (the electrolyte is considered symmetric).

As already indicated above, even if there are diffusion and migration of ions (no supporting electrolyte), the transport problem can be mathematically reduced to a simple diffusion process (transport in a binary electrolyte). ${ }^{15,24}$ The grain growth can therefore be considered as diffusion-limited. Furthermore, this diffusion process is quasi-stationary because the concentration field adapts sufficiently rapidly to the change in grain size. This is directly related to the strong difference in metal density between the grain (solid metal) and the liquid (dissolved metal salt), see also the discussion in Ref. 42. As a consequence, the Laplace equation is satisfied in the liquid: 
a) $33 \mathrm{~mA} / \mathrm{cm}^{2}$

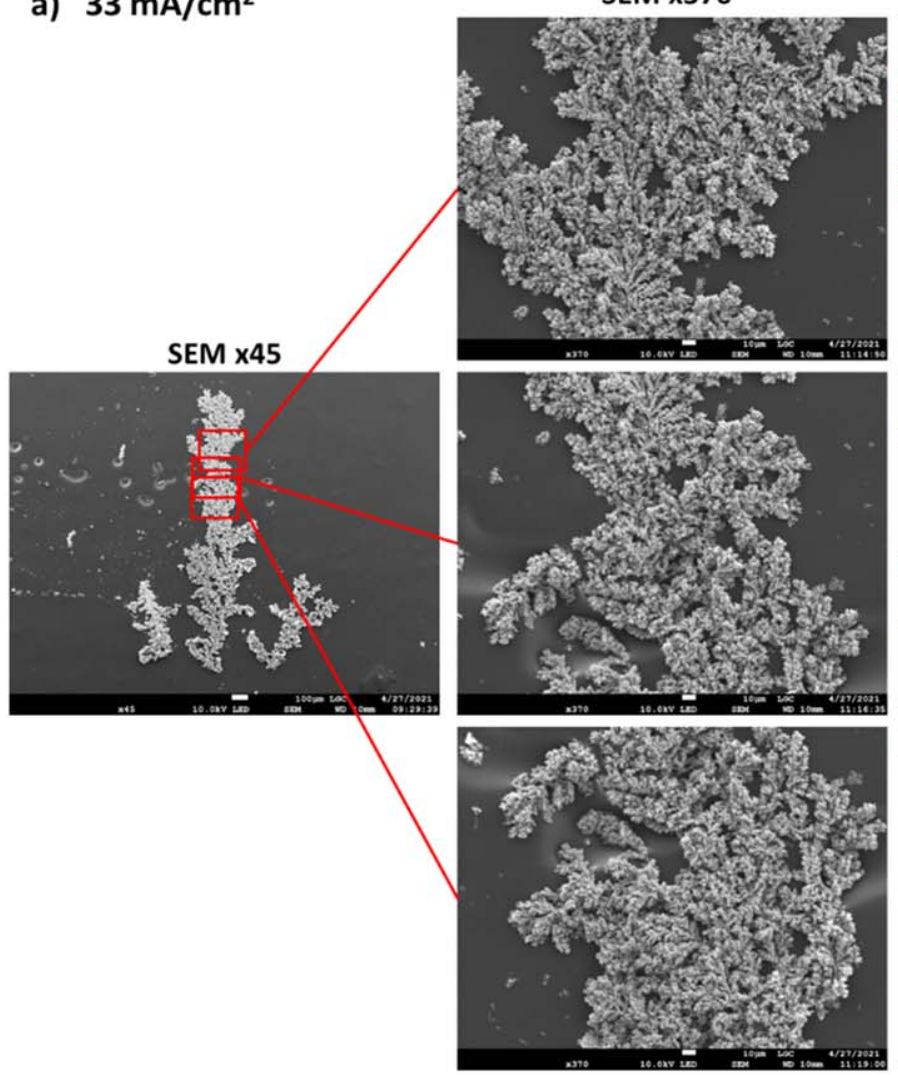

b) $266 \mathrm{~mA} / \mathrm{cm}^{2}$

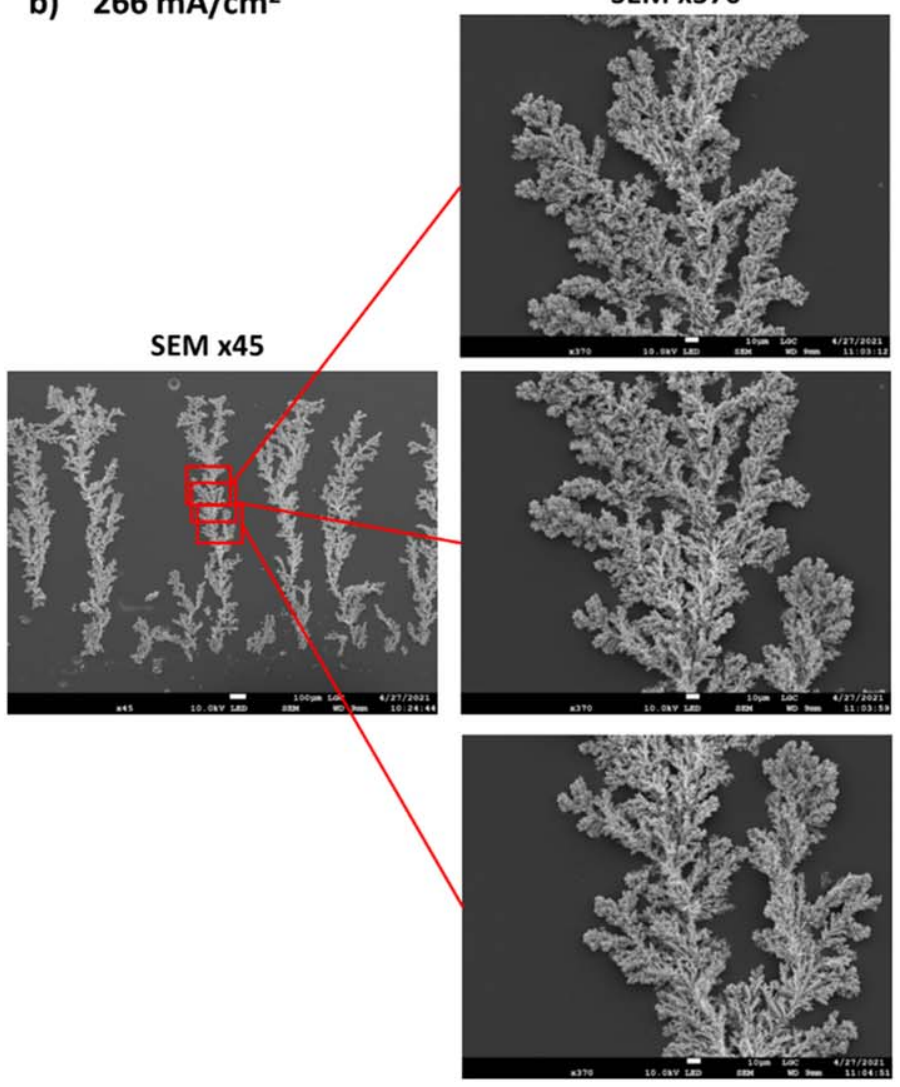

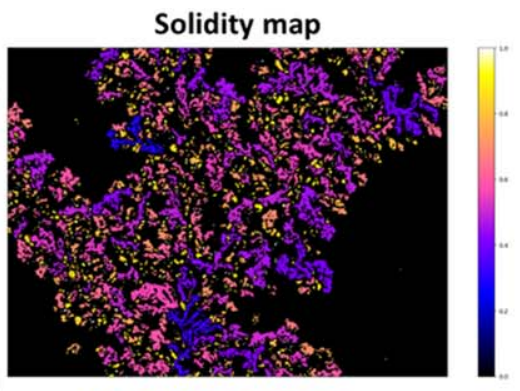
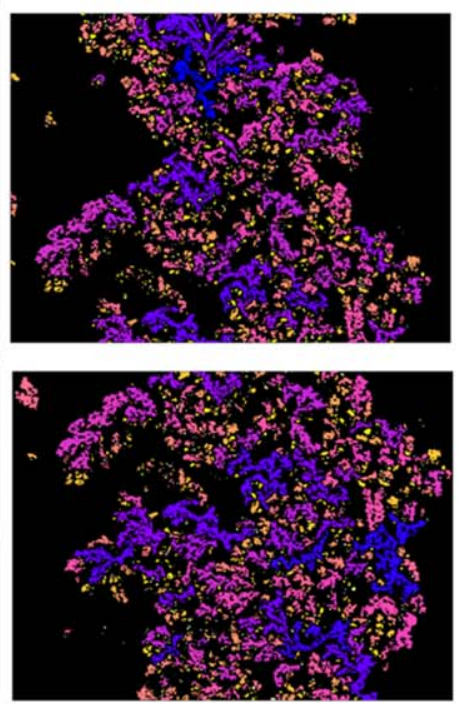

Solidity map
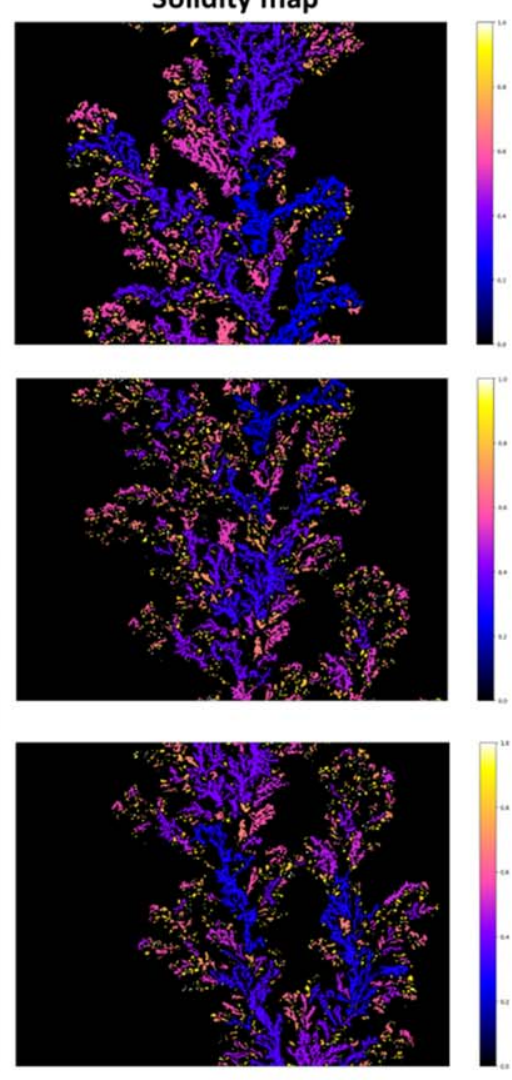

Figure 11. SEM images of copper branches over a significant part of their length at $\times 370$ magnification and the corresponding solidity maps, for $33 \mathrm{~mA} \mathrm{~cm}^{-2}$ in (a) and $266 \mathrm{~mA} \mathrm{~cm}^{-2}$ in (b); $c_{0}=0.5 \mathrm{M}$. Solidity maps are obtained by image processing using python: automatic thresholding from MaxEntropy method (Kapur, pythreshold), particle detection and morphology properties (skimage). 


\section{$0.25 \mathrm{M}$}


$0.75 \mathrm{M}$
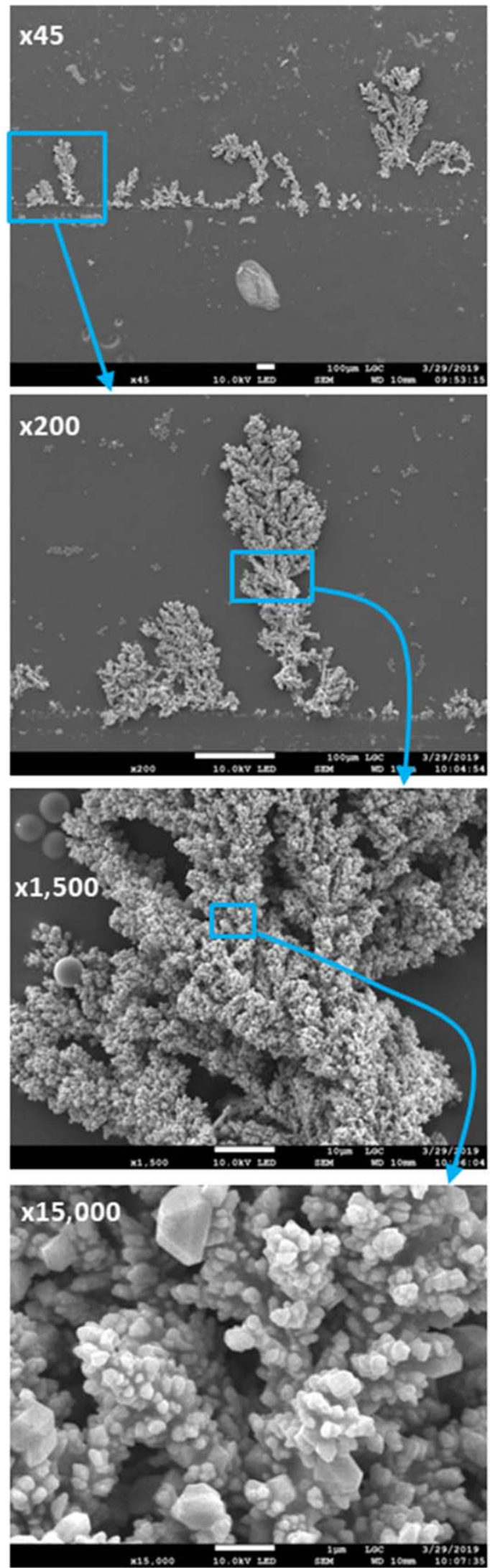

Figure 12. SEM images of copper branches, for two concentrations $c_{0}$ of 0.25 and $0.75 \mathrm{M}$, and for a current density $j$ of $66 \mathrm{~mA} \mathrm{~cm}$. 

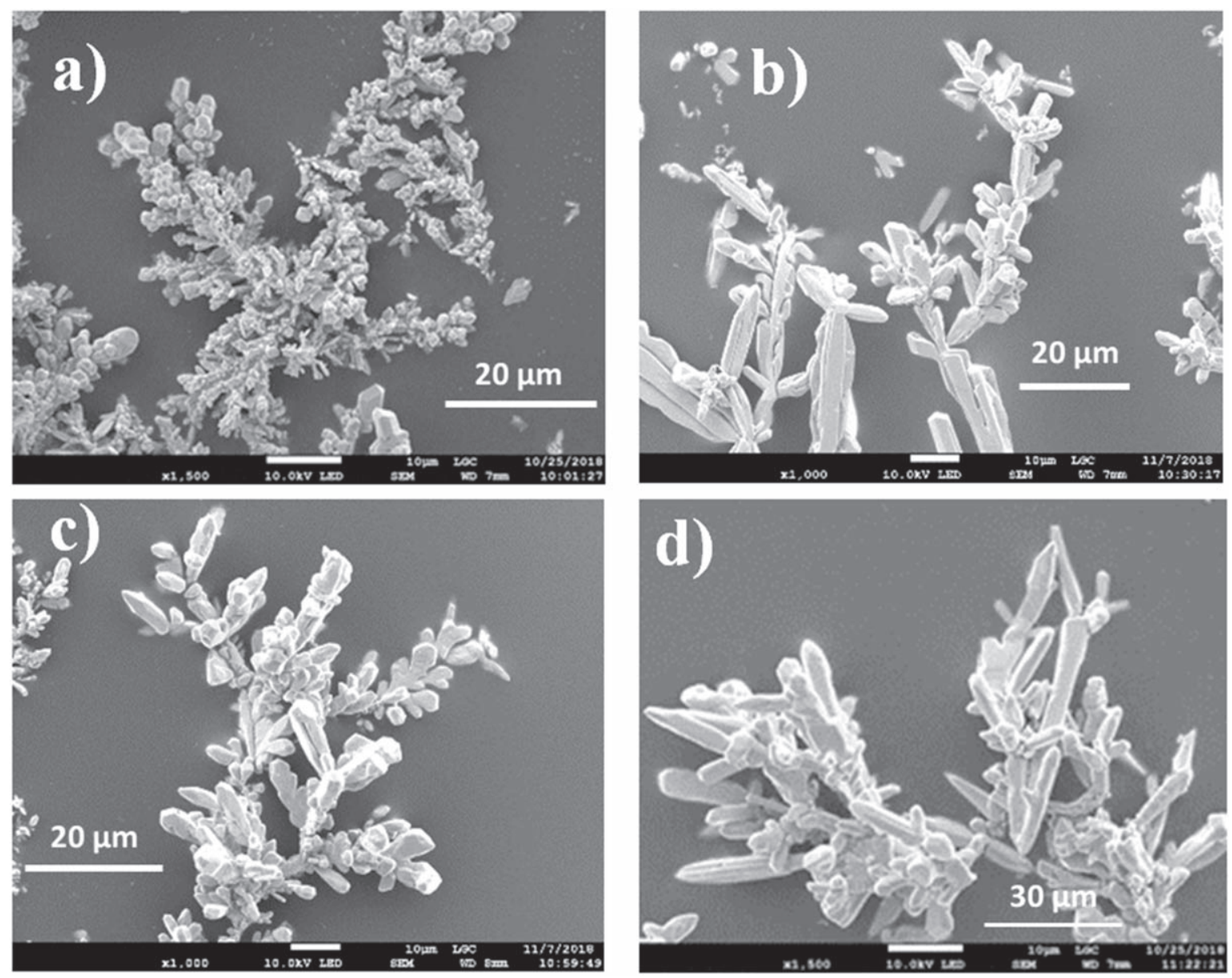

Figure 13. SEM images of silver branches (top of the branches) for the following operating conditions: $\left[\mathrm{AgNO}_{3}\right]=c_{0}=0.25 \mathrm{M}$, and $j=33 \mathrm{~mA} \mathrm{~cm}^{-2}$ (a), $j=$ $66 \mathrm{~mA} \mathrm{~cm}^{-2}$ (b), $j=133 \mathrm{~mA} \mathrm{~cm}^{-2}$ (c) and $j=266 \mathrm{~mA} \mathrm{~cm}^{-2}$ (d).

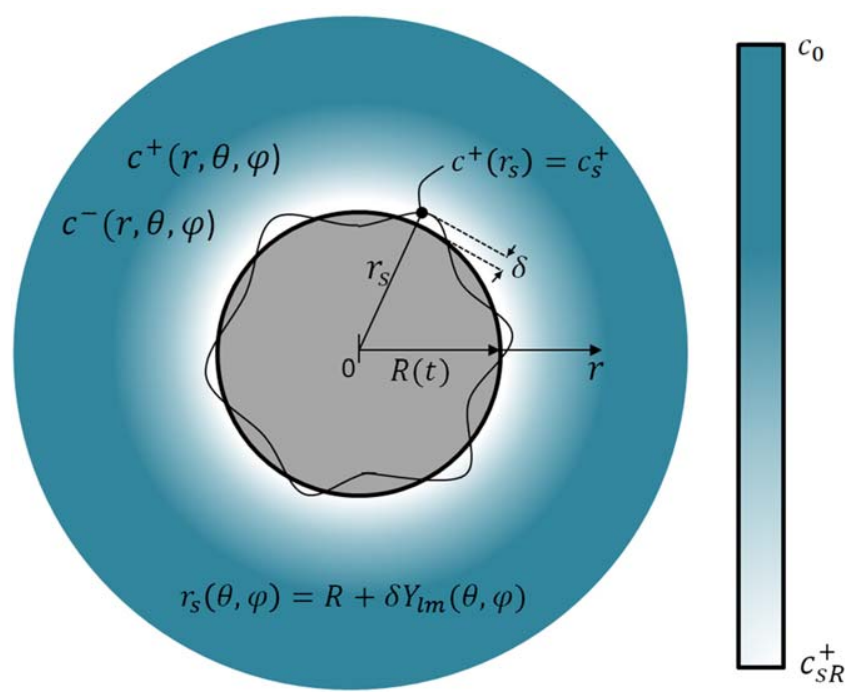

Figure 14. Sketch of the growth of a spherical grain (dark grey), surrounded by an electrically neutral and diffusional region (color map), and subject to shape deformations whose the amplitude is measured by $\delta(t)$.

$$
\nabla^{2} c^{+}=0
$$

The cation concentration at the grain surface $c_{s}^{+}$depends on both the overpotential $\eta$ and the local curvature $K$ :

$$
\eta(t)=\frac{\mathrm{RT}}{z \mathrm{~F}} \log \left(\frac{c_{s}^{+}(t)}{c_{0}}\right)-\frac{\gamma_{P L} V_{m}}{z \mathrm{~F}} K(t),
$$

where $R$ is the ideal gas constant, $T$ the temperature, $\gamma_{P L}$ is the free surface energy of the interface between the grain and the liquid, $V_{m}$ the molar volume and $K$ the local curvature of the interface. In the right hand side of this latter equation, the first term corresponds to the concentration overpotential ${ }^{53}$ and the second one is a correction to account for the curvature of the interface (Gibbs-Thomson effect) ${ }^{38,54}$ for a spherical grain of radius $R, K=2 / R$. In accordance with the diffusion-limited growth assumption, the activation overpotential, related to the electrochemical kinetics, is neglected.

After linearization assuming small $K$ in Eq. $4, c_{s}^{+}$depends on shape deformations according to the following relation:

$$
c_{s}^{+}=c_{s f}^{+}(1+\Gamma K),
$$

where $c_{s f}^{+}=c_{0} \exp \left(\frac{z F}{\mathrm{RT}} \eta(t)\right)$ is the equilibrium concentration of the cations at a flat metal surface and $\Gamma=\frac{\gamma_{P L} V_{m}}{\mathrm{RT}}$ the capillary length.

As in classical shape stability analyses, the deformations are modeled with spherical harmonics $Y_{l m}(\theta, \varphi)$ of degree $l$ and mode $m$ ( $\theta$ and $\varphi$ being the angular spherical coordinates). The location of the grain surface $r_{s}$ is given by (Fig. 14):

$$
r_{s}(t, \theta, \varphi)=R(t)+\delta(t) Y_{l m}(\theta, \varphi)
$$

where $\delta$ is the deformation amplitude. We aim to find from which grain radius $R$, the amplification rate $\dot{\delta} / \delta$ becomes positive. 
To the first order in $\delta$, the solution of the Laplace equation (Eq. 3) satisfying the boundary condition Eq. 5 is given by (by identification with Eq. 7 in Ref. 42):

$$
\begin{aligned}
& c^{+}(r, \theta, \varphi)=\frac{\left(c_{s f}^{+}-c_{0}\right) R+2 c_{s f}^{+} \Gamma}{r}+c_{0} \\
& +\frac{\left(c_{s f}^{+}-c_{0}\right) R^{l}+c_{s f}^{+} \Gamma R^{l-1} l(l+1)}{r^{l+1}} \delta Y_{l m}(\theta, \varphi) .
\end{aligned}
$$

The conservation of the deposited metal at the grain surface leads to:

$$
v \rho=\left.\frac{D}{1-t_{c}} \frac{\partial c^{+}}{\partial r}\right|_{r_{s}}+\left.\frac{v}{1-t_{c}} c^{+}\right|_{r_{s}}
$$

where $\rho$ is the metal density $\left(\mathrm{mol} \mathrm{m} \mathrm{m}^{-3}\right), D$ the mean diffusion coefficient defined by $D=\left(u_{c} D_{a}+u_{a} D_{c}\right) /\left(u_{c}+u_{a}\right)$ (where $D_{a}$ and $D_{c}$ are the diffusion coefficients of anions and cations respectively), and $v=d r_{s} / d t$ the normal velocity of the surface. ${ }^{42}$

Combining Eqs. 7 and 8, the interface velocity expresses as (Eq. 8 in Ref. 42):

$$
\begin{aligned}
\dot{R}+\dot{\delta} & =\frac{D}{\rho\left(1-t_{c}\right)-c_{s R}^{+}}\left[\frac{c_{0}-c_{s R}^{+}}{R}+\frac{\delta Y_{l m}}{R}\right. \\
& \left.\times\left((l-1) \frac{c_{0}-c_{s f}^{+}}{R}-\frac{\Gamma}{R^{2}} c_{s f}^{+}\left(l(l+1)^{2}-4\right)\right)\right],
\end{aligned}
$$

where $c_{s R}^{+}=c_{s f}^{+}(1+2 \Gamma / R)$. In the right hand side of Eq. 9 , the term in square brackets is exactly the same as in Ref. 42. Mullins \& Sekerka show that shape instability requires that the deformations grow faster than the grain expansion, $(\dot{\delta} / \delta) /(\dot{R} / R)>1$, and $l \geqslant 3$. This leads to the definition of the threshold radius $R_{t}$ :

$$
R_{t}=42 \Gamma \frac{c_{s f}^{+}}{c_{0}-c_{s f}^{+}} .
$$

Shape deformations appear if $R$ exceeds $R_{t}$. Contrary to usual phase changes (crystallization, condensation, etc.), where the surface concentration is constant (equilibrium saturation), here, $c_{s f}^{+}$changes during the grain growth because of its dependence on the overpotential, $c_{s f}^{+}=c_{0} \exp ((z F / \mathrm{RT}) \eta)(\mathrm{Eq} .5)$. Consequently, the stability condition $\left(R_{t}\right)$ changes during the grain growth.

The periodic temporal evolution of $\eta$ is sketched in Fig. 15b and explained here. According to Fleury's nucleation/growth model, just after the nucleation of a new grain, the incoming current is concentrated on its surface to make it grow at a constant growth rate (the previous grain does no longer growth, Fig. 15a); the grain radius thus follows a one-third power law on a time period of $T$, Fig. 15a.

Initially, the grain size $R(0)$ is very low, typically $\sim 1 \mathrm{~nm}$ (corresponding to overpotential $\sim 100 \mathrm{mV}$ ). This induces a so high interface velocity $\left(\propto R^{-2}\right)$ that both space charge and electrolyte depletion do not have time to develop (as initially postulated by Fleury ${ }^{39}$ ) and this prevents $c_{s}^{+} / c_{0}$ to reach small values. At the early stage of the grain growth, the Gibbs-Thomson overpotential then overcomes the concentration overpotential $\eta(t) \approx-\frac{\gamma_{P L} V_{m}}{z F} \frac{1}{R(t)}$. This induces a fast decrease of $\eta$ at the beginning of a cycle, Fig. 15b.

For longer times, up to the end of grain growth, the interface velocity is well lowered (because the grain is now large, $R \rightarrow R_{g} \sim$ 100 nm, Figs. 8-10 and 12) and both electrolyte depletion and space charge have time to develop. ${ }^{39}$ This leads to $c_{s}^{+} / c_{0} \ll 1$ and the overpotential now corresponds to the concentration overpotential $\eta(t) \approx \frac{\mathrm{RT}}{z F} \log \left(\frac{c_{s}^{+}(t)}{c_{0}}\right)$. As it is shown below, for this regime, $c_{s}^{+}$is proportional to $j_{g}(t)^{2 / 3}$ (see also Eqs. A.21 with A.19 and A.20), with $j_{g}(t)$ the current density on the growing grain surface. The assumption of constant growth rate induces $j_{g} \propto R^{-2}$ and consequently $\eta(t) \approx C-\frac{4}{3} \log (R(t))$ (where $C$ is a constant) as sketched in Fig. 15b. When $\eta$ reaches a critical value $\eta_{0}=\eta(0)=\eta(T)$, a new grain nucleates and grows immediately on the one that was growing and a new cycle starts, Fig. 15a. Note that the temporal signal of $\eta$ is very similar to the temporal signal of the surface electric field shown in Ref. 39 (Fig. 4).

The corresponding signal of $R_{t}$ is plotted in Fig. 15c. On a growth cycle, the instability is favored at the end of grain growth. This differs from usual situations where $R_{t}$ is constant. As sketched in Figs. $15 \mathrm{~d}-15 \mathrm{e}$, the stability condition for a growing grain to be stable on a full cycle is $R_{g}<\min \left(R_{t}\right)$.

The estimation of $\min \left(R_{t}\right)$ requires $\eta_{0}$ (Eq. 10) which can be derived at the end of a grain growth. For these long growth times, the electrolyte depletion at the electrode surface induces a divergent electric field (assuming electroneutrality). By considering the corresponding Poisson-Nernst-Planck problem, assuming a stationary and one-dimensional system, Chazalviel ${ }^{14}$ showed the very high electric field at the electrode surface leads to the formation of a space charge region. The electrolyte phase consists of two regions: $:^{14,39}$ in the vicinity of the electrode (grain) surface, on a certain small thickness $x_{I}(\sim 100 \mathrm{~nm})$, the solution is not electrically neutral, $c^{+} \gg c^{-}$, this is the charge space region, while beyond $x_{I}$, the solution is neutral $\left(c^{+}=c^{-}\right)$. In the space charge region, both cation concentration and electric potential profiles can be derived according to Chazalviel's model. ${ }^{14}$ This gives access to $c_{s}^{+}(t=T)$ and therefore to $\eta_{0} \approx \frac{\mathrm{RT}}{z F} \log \left(\frac{c_{s}^{+}(T)}{c_{0}}\right)$.

Note that the use of Chazalviel's model requires the system to be stationary. This is actually the case for long growth times for which grain grow so slowly that mass transport, across the space charge by both diffusion and migration, can be considered as quasi-stationary. This is justified by the estimation of the characteristic growth time $T=2 R_{g} / v_{g}$, the characteristic diffusion time $T_{d}=x_{I}{ }^{2} / D_{c}$ and the characteristic migration time $T_{m}=x_{I} /\left(z F\left|E_{s}\right| D_{c} /(\mathrm{RT})\right)$. By estimating $x_{I}$ and $\left|E_{s}\right|$ from the stationary and one-dimensional Chazalviel's theory (Eq. 23, 25 and 27 in Ref. 14 and considering a potential drop across the space charge region, $\left|E_{S}\right| x_{I}$, of $1 \mathrm{~V}$, Appendix B) and considering the typical encountered ranges for $R_{g}$ $[150,550 \mathrm{~nm}]$ and $v_{g}\left[1,20 \mu \mathrm{m} \mathrm{s}^{-1}\right]$ (for $j \in\left[33,100 \mathrm{~mA} \mathrm{~cm}{ }^{-2}\right]$ and $c_{0} \in[0.50,0.75 \mathrm{M}]$, Fig. 7$), T_{d} / T$ and $T_{m} / T$ are lower than $0.07 \%$. This greatly simplifies the modeling contrary to the beginning of a grain growth for which the problem is transient and requires numerical simulation. ${ }^{39}$

Nevertheless, $x_{I}$, computed from Chazalviel's model, which is one-dimensional, $\left(x_{I} \sim 100 \mathrm{~nm}\right)$, is of the same order of magnitude as the size of the grains $(\sim 100 \mathrm{~nm}$, Figs. $8-9)$. Consequently, we expect that $x_{I}$ depends on the grain radius.

Here, the Chazalviel's model is revisited taking into account a spherical geometry (Appendix A). For a spherical electrode (grain) of radius $R$ subject to a current density $j_{g}$, from the theoretical framework of Chazalviel, ${ }^{14}$ we obtain the following profiles across the space charge layer for both electric field and cation concentration:

$$
\phi\left(x_{I}+R\right)-\phi(r)=R \sqrt{\frac{2 j_{g}}{3 z \mathrm{~F} u_{c} \varepsilon \varepsilon_{0}}} \int_{r}^{x_{I}+R} \frac{\sqrt{\left(x_{I}+R\right)^{3}-x^{3}}}{x^{2}} d x,
$$


a)

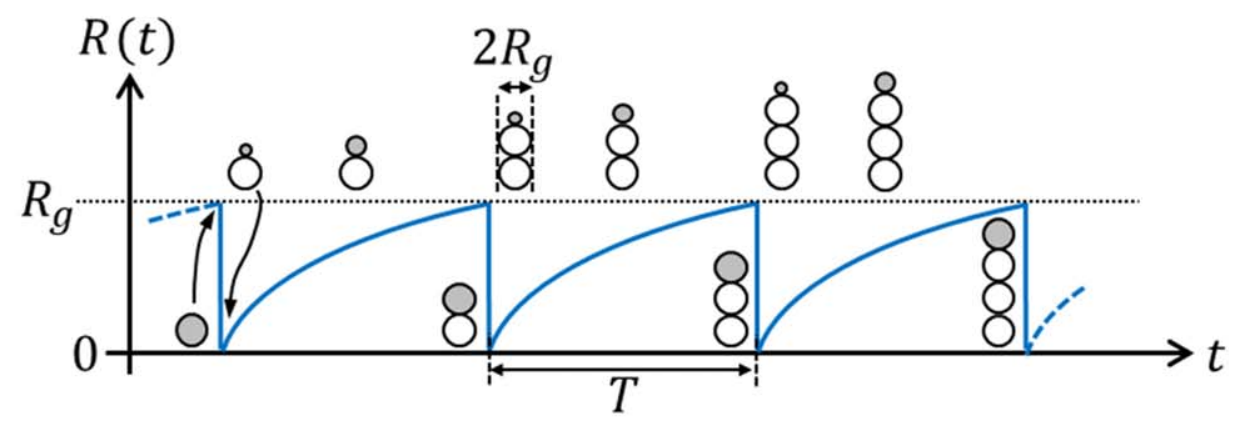

b)

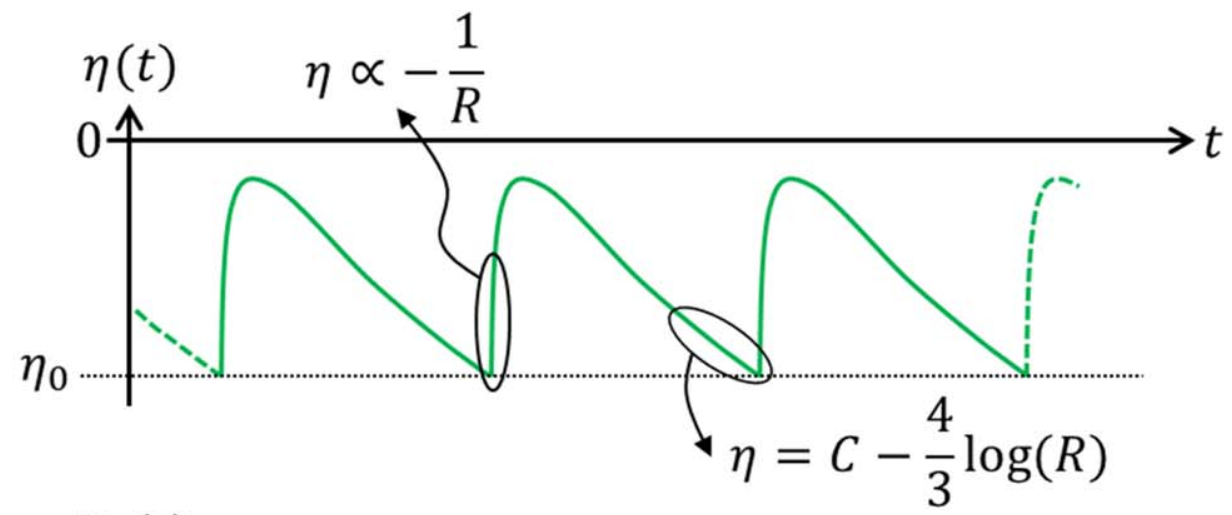

c)

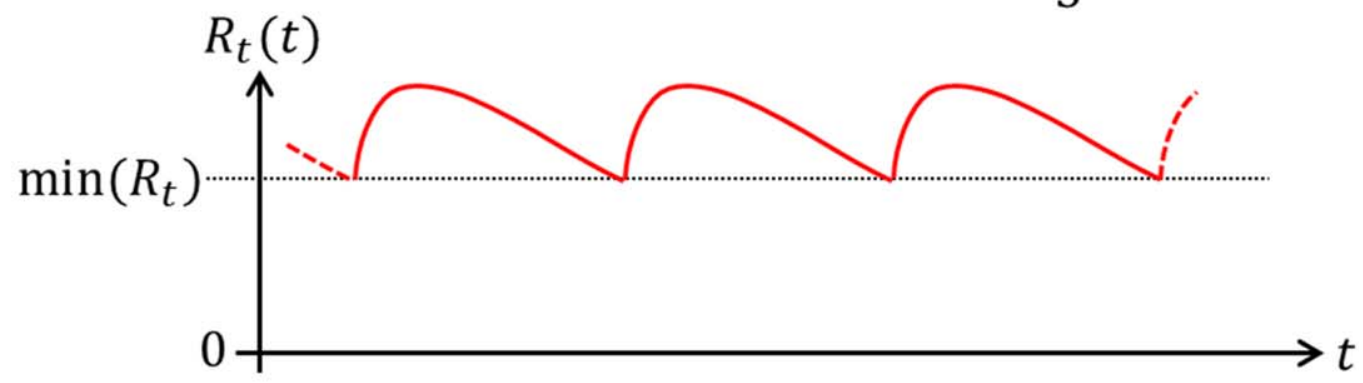

d)

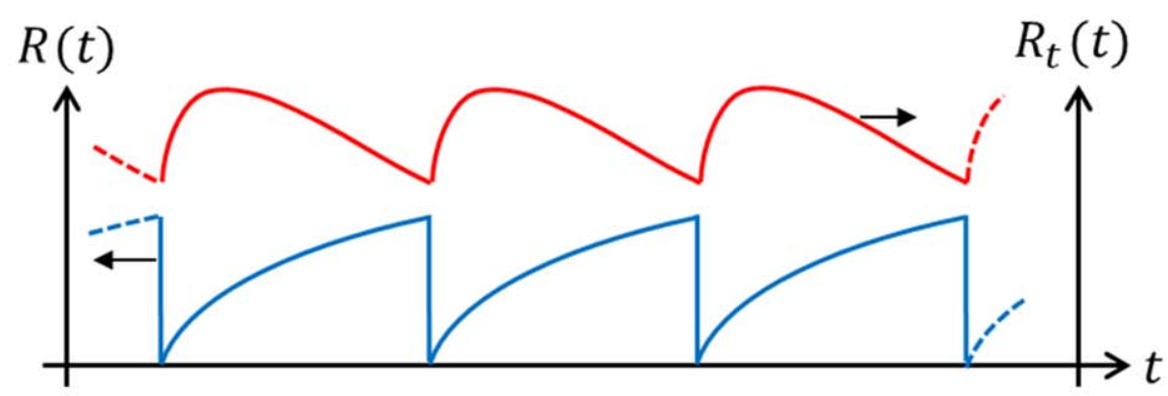

e)

Stable grain $R_{g}<\min \left(R_{t}\right)$
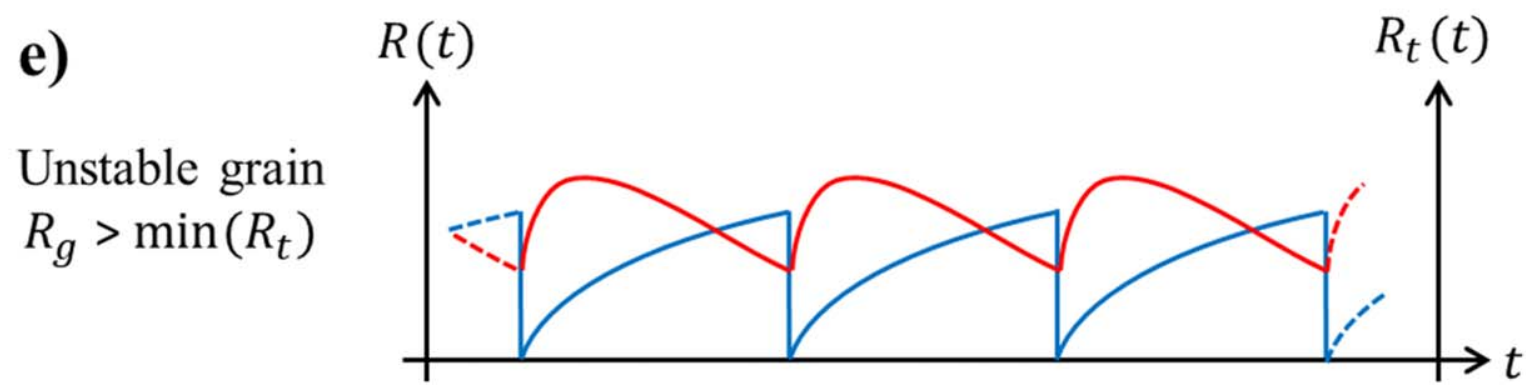

Figure 15. Sketches of the periodic temporal evolution of radius of a growing grain $R$ (a), overpotential $\eta$ (b) and threshold radius for the onset of shape instability assuming a diffusion-limited growth $R_{t}$ given by Eq. 10 (c). In (a), successive nucleation/growth events are sketched, the top grain, colored in gray, is the grain which is growing. The $R(t)$ and $R_{t}(t)$ signals are plotted in the same graph for both cases stable grain (d) and unstable grain (e). 


$$
c^{+}(r)=\left[\frac{2(z \mathrm{~F})^{3} u_{c}}{3 j_{g} \varepsilon \varepsilon_{0}}\left(\frac{\left(x_{I}+R\right)^{3}-r^{3}}{R^{2}}\right)\right]^{-1 / 2},
$$

for $R+x_{I} \geqslant r \geqslant R$ and where $\varepsilon_{0}$ is the vacuum permittivity, $\varepsilon$ the relative permittivity of water and $r$ the radial coordinate. The potential drop across the space charge layer $\delta V$ is obtained from Eq. $11\left(\delta V=\phi\left(x_{I}+R\right)-\phi(R)\right)$ :

$$
\delta V=R \sqrt{\frac{2 j_{g}}{3 z \mathrm{~F} u_{c} \varepsilon \varepsilon_{0}}} \int_{R}^{x_{I}+R} \frac{\sqrt{\left(x_{I}+R\right)^{3}-x^{3}}}{x^{2}} d x .
$$

$\delta V$ has been measured and it is around $1 \mathrm{~V}$ for each case (Appendix B). This is in agreement with similar measurements. ${ }^{41,45}$

The link between $j_{g}$ and the operating parameters $\left(j\right.$ and $\left.c_{0}\right)$ can be established from Fleury's nucleation/growth model. An important statement of this model is the link between microstructure and macro-scale (pattern) through the relation: ${ }^{39}$

$$
T=\frac{2 R_{g}}{v_{g}},
$$

Note that Eq. 14 is obtained by neglecting the size of the nucleus $R(0)$ in front of $R(T)=R_{g}$. According to Fleury's nucleation/growth model, ${ }^{39}$ the grain growth rate is constant and the Faraday's law leads to:

$$
\frac{I_{g}}{z F}=\frac{d}{d t}\left(\frac{4}{3} \pi \rho R^{3}\right)
$$

where $I_{g}=\left(4 \pi R^{2}\right) j_{g}$ is the incoming current on one growing grain. After integration of Eq. 15, coupled with Eqs. 2 and 14, we obtain a relation for the current density at the end of the growth phase $j_{g}(T)$ :

$$
j_{g}(T)=\frac{1}{6} z \mathrm{~F} \rho v_{g}
$$

Equation 16 is an important relation because it gives a direct access to the current density on the grain surface at the end of growth, as a function of the operating parameters through $v_{g}$ (Eq. 2) whatever the arrangement of the branches on the pattern scale.

For each set of input parameters $\left(j, c_{0}, R=R_{g}\right)$, the concentration $c_{s f}^{+}$, which is equal to the surface concentration $c_{s}^{+}(T)=c^{+}\left(R_{g}\right)$, can therefore be estimated combining Eqs. 2, 12, 13 and 16. Note that $x_{I}$ is determined by numerically solving Eq. 12, jointly using a nonlinear solver and a numerical integration; $x_{I}$ is found to have a very weak dependence on $R$ unlike $c_{s}^{+}(T)$ (Appendix A). The estimated values of $c_{s f}^{+}$are so low (maximum value of $10^{-3} \mathrm{M}$, Appendix A, Fig. A.1c) that the growing grains are expected to be always unstable. The prefactor of $\delta$ in the second member of the right hand side of Eq. 9 is always positive. The damping of shape deformations ( $\dot{\delta} / \delta<0$, in Eq. 9) would require prohibitive values of $l(>100)$ for the typical grain size $R$ of $100 \mathrm{~nm}: R_{g} \gg \min \left(R_{t}\right)$. This is not consistent with the experiments which clearly show that there is a stable growth regime of the grains.

Furthermore, it is interesting to analyze the variation of $\min \left(R_{t}\right)$ with $j$ and $c_{0}$. According to Eq. $10, \min \left(R_{t}\right) \approx 42 \Gamma c_{s}^{+}(T) / c_{0}$. The value of $c_{s}^{+}(T)$ results from the specific interaction between both cation concentration and electric potential, in the space charge layer. Indeed, across the space charge layer, the current flow is mainly ensured by the migration of cations (Appendix A, Eq. A.15), $\left((z \mathrm{~F})^{2} u_{c}\right) \delta V c_{s}^{+}(T) / x_{I} \approx j_{g}(T)$. Since the potential drop $\delta V$ is constant and that $j_{g}(T) \propto j / c_{0}$ (Eqs. 13 and 16), $c_{s}^{+}(T) / x_{I}$ is found to be proportional to $j / c_{0}$. By considering the Poisson equation (Eq. A.14), $\delta V / x_{I}{ }^{2} \approx\left(z F /\left(\varepsilon \varepsilon_{0}\right)\right) c_{s}^{+}(T)$, the following power laws are obtained:
$x_{I} \propto\left(j / c_{0}\right)^{-1 / 3}$ and $c_{s}^{+}(T) \propto\left(j / c_{0}\right)^{2 / 3}$. If $j / c_{0}$ increases (i.e. $v_{g}$ increases), this induces an increase of the electric field $\left(\approx \delta V / x_{I}\right)$ which is limited, to avoid divergence, by the enrichment in cations, $c_{s}^{+}(T)$ increases.

Consequently, $\min \left(R_{t}\right)$ increases with $j\left(\min \left(R_{t}\right) \propto j^{2 / 3}\right)$ that is also not consistent because this would favor stability instead of instability when $j$ is increased. Additionally, $\min \left(R_{t}\right)$ decreases with the concentration $c_{0}\left(\min \left(R_{t}\right) \propto c_{0}{ }^{-5 / 3}\right)$. This would suggest that $j_{c}$ decreases with $c_{0}$. This trend is also not correlated with the experimental results.

These differences show that the standard shape stability analysis, adapted from Mullins \& Sekerka model, is not able to describe the appearance of dendrites on the level of branch microstructure. This probably comes from the too simplified model of mass transport which considers only diffusion. Indeed, in the vicinity of the grain surface, due to the presence of the space charge layer, the mass transport problem can no longer be assimilated to a diffusion situation. In the space charge layer, the migration overcomes the diffusion of cations $\left({ }^{14}\right.$ and Appendix A) and this affects the stability condition.

Effect of the space charge layer on the stability threshold.-Even if a full derivation is required (as in Ref. 52 but at the scale of a grain and for a spherical shape, this will be done in another work), we could predict the main effect of the space charge on the shape stability.

By considering the limitation by migration of the cations, Eq. 8 becomes:

$$
v=\frac{z u_{c} \mathrm{~F}}{\rho-\left.c^{+}\right|_{r_{s}}}\left[c^{+} \frac{\partial \phi}{\partial r}\right]_{r_{s}} .
$$

In the space charge layer, the amplitude of the variation of $\phi$ is $\sim \delta V$, on a length of about $x_{I}$ and therefore $\left.\frac{\partial \phi}{\partial r}\right|_{r_{s}} \approx \delta V / x_{I}$. As in diffusionlimited cases, there is therefore a destabilizing point effect but induced by the electric potential across a shell (gradient zone) which the thickness is $x_{I}$; this differs significantly from diffusion-limited cases where the shell stays conform to the grain during its growth $\left.\frac{\partial c^{+}}{\partial r}\right|_{r_{s}} \approx c_{0} / R$.

During the growth of a grain, $x_{I} \propto j_{g}^{-1 / 3}$ (Eq. A.19), and therefore $x_{I} \propto t^{2 / 9}$. For short times, this power law induces a faster increase for $x_{I}$ than for $R \propto t^{1 / 3}$ (shell thickness for diffusion-limited cases). For long times, $t \rightarrow T, x_{I}$ reaches typical values around $100 \mathrm{~nm}$ and it varies rather slowly with time $\left(t^{2 / 9}\right)$. This prevents the amplification of shape deformations $\left(\delta \ll x_{I}\right)$ as long as the grain size $R(t)$ is lower than $\sim x_{I}(T) \sim 100 \mathrm{~nm}$; the grain grows inside a shell with an almost constant thickness. On the contrary, for a diffusion situation, the point-like effect is likely to appear earlier, for smaller grains, and this leads to a low instability threshold as obtained with Eq. 10 (where the oversaturation $\left(c_{0}-c_{s f}^{+}\right) / c_{s f}^{+}$is typically high in such an electrochemical situation). The taking into account of a space charge must therefore lead to a positive instability threshold which the order of magnitude is the same as the one of $x_{I}(T) \sim 100 \mathrm{~nm}$. This latter is compatible with the actual size of the grains encountered here $R_{g}$ which is also $\sim 100 \mathrm{~nm}$ (Figs. 8-9). Furthermore, since $x_{I}(T) \propto\left(j / c_{0}\right)^{-1 / 3}$, this suggests that the onset of shape instability, and thus the presence of dendrites, is favored when $j$ is increased and when $c_{0}$ is decreased. These predicted tendencies are now consistent with the experiments.

\section{Conclusions}

It is experimentally shown that the transition from non-adherent ramified branches (nucleation/growth regime) towards dendrites (growth regime) appears at the scale of the grains constituting the 
ramified branches. These non-dendritic crystals undergo a shape instability leading to the formation of dendrites when $j$ exceeds a given critical applied current density. This latter increases with the electrolyte concentration $c_{0}$.

The fact that the grains become unstable when $j$ increases could appear usual (similarly to other crystallization or condensation phenomena) but this is not so simple. Notably because both the grain size and the oversaturation (Eqs. 10 and A.21) decrease with the ratio $j / c_{0}$ that generally prevents shape instability and thus dendritic growth. According to the standard Mullins \& Sekerka shape stability analysis (Eq. 10), unlike the experiment results, these tendencies suggest an enhanced shape stability when $j / c_{0}$ increases. This discrepancy is due to the periodic formation of a space charge layer (which avoids the divergence of the electric field, a specificity of the present situation) which strongly modifies the transport problem, compared to classic (diffusion-limited) crystallization or condensation situations.

By considering a space charge layer around a growing grain, the right tendencies are qualitatively obtained. It was previously demonstrated that the space charge region plays a major role on the formation of ramified branches (onset of branch growth ${ }^{14}$ and re-nucleation process ${ }^{39}$ ). Here, the obtained results suggest the space charge region also plays a role in:

- stabilization of the shape of grains constituting ramified branches (the dendritic growth is delayed)

- onset of dendritic growth at the scale of the growing grains.

This shows, once again, ${ }^{39}$ the originality of this growth phenomenon compared to other solidification or condensation situations.

This study focuses only on the onset of dendritic growth. Many questions remain open especially beyond the dendritic transition, when the branch microstructure is mixed (grain assemblies and dendritic crystals). For example, in the case of copper (as here) and iron $\left({ }^{11,55}\right)$, why the size of the dendrites is always so low that, on the macro-scale, the pattern remains ramified? While for zinc (and in some extent for silver), very large dendrites are obtained. ${ }^{17}$ There should be a competition between re-nucleation process and dendrite growth (beyond shape instability) governed by material (anisotropic) properties and mass transport at length scales of both macroscale and branch microstructure.

The obtained improved knowledge on the transition between non-dendritic and dendritic regimes will favor the development of an alternative synthesis of metal nanomaterial based on the exploitation of the nanostructure of the branches.

\section{Acknowledgments}

This study was supported in part by the French MSR Graduate Research Fellowship and the authors would like to thank the Paul Sabatier University for funding the research. This study was supported in part by the program PHC Tournesol FR $N^{\circ} 38278$ NE. We thank Pierre Colinet for valuable discussions. We are very grateful to M. L. de Solan-Bethmale (Laboratoire de Génie Chimique) and S. Le Blond du Plouy (Centre de microcaractérisation Raimond Castaing) for SEM observations.

\section{Appendix A. Derivation of the Relations of the Space Charge Region for a Spherical Particle}

The electrochemical growth of a spherical particle of radius $R(t)$ is considered. The derivation is carried out for the end of the growth $\left(t \rightarrow T, R(t) \rightarrow R_{g}\right.$ ) when the system can be considered quasistationary (see the section related to the derivation of the threshold grain size). In the liquid, $r \geqslant R(t)$, the concentration of cations and anions and the electric potential satisfy the transport equations:

$$
z u_{c} \mathrm{~F} \nabla \cdot\left(c^{+} \nabla \phi\right)+D_{c} \nabla^{2} c^{+}=0,
$$

$$
-z u_{a} \mathrm{~F} \nabla \cdot\left(c^{-} \nabla \phi\right)+D_{a} \nabla^{2} c^{-}=0,
$$

as well as Poisson equation:

$$
\nabla^{2} \phi=-\frac{\mathrm{F}}{\varepsilon \varepsilon_{0}}\left(z c^{+}-z c^{-}\right) .
$$

There are no advection terms since they are typically negligible for such small particles (the Péclet number $\sim v_{g} R_{g} / D$ is lower than 0.01). Using spherical coordinates, the relations A1-3 become:

$$
\begin{gathered}
D_{c} \frac{d}{d r}\left(r^{2} \frac{d c^{+}}{d r}\right)+z u_{c} \mathrm{~F} \frac{d}{d r}\left(r^{2} c^{+} \frac{d \phi}{d r}\right)=0, \\
D_{a} \frac{d}{d r}\left(r^{2} \frac{d c^{-}}{d r}\right)-z u_{a} \mathrm{~F} \frac{d}{d r}\left(r^{2} c^{-} \frac{d \phi}{d r}\right)=0, \\
\frac{1}{r^{2}} \frac{d}{d r}\left(r^{2} \frac{d \phi}{d r}\right)=-\frac{\mathrm{F}}{\varepsilon \varepsilon_{0}}\left(z c^{+}-z c^{-}\right) .
\end{gathered}
$$

Integrating Eq. A.4 between $R$ and $r \geqslant R$, considering the total mass flux of cations is $4 \pi R^{2}\left(j_{g} /(z \mathrm{~F})\right)$ at the particle surface, leads to:

$$
D_{c} \frac{d c^{+}}{d r}+z u_{c} \mathrm{~F} c^{+} \frac{d \phi}{d r}=\frac{j_{g}}{z \mathrm{~F}}\left(\frac{R}{r}\right)^{2} .
$$

By doing the same for the anions (Eq. A.5), considering the total mass flux of anions is 0 at the particle surface, leads to:

$$
D_{a} \frac{d c^{-}}{d r}-z u_{a} \mathrm{Fc}^{-} \frac{d \phi}{d r}=0 .
$$

Integrating Eq. A.6 between $r \geqslant R$ and a distance $L^{\prime}$ being sufficiently high such that the electrolyte concentration $\approx c_{0}$, we obtain:

$$
c^{-}(r)=c_{0} \exp \left(-\frac{z u_{a} \mathrm{~F}}{D_{a}}\left(\phi\left(L^{\prime}\right)-\phi(r)\right)\right)
$$

Combining Eqs. A.7 and A.8, assuming electroneutrality $\left(c^{-}=c^{+}\right)$ and using $u_{c}=D_{c} / \mathrm{RT}$ and $u_{a}=D_{a} / \mathrm{RT}$, we obtain:

$$
2 D_{c} \frac{d c^{+}}{d r}=\frac{j_{g}}{z \mathrm{~F}}\left(\frac{R}{r}\right)^{2} \text {. }
$$

Following the theoretical model of Chazalviel, ${ }^{14}$ the thickness of the charge region $x_{I}$ is defined such that, for $r>R+x_{I}$, the electroneutrality applies, and, for $R \leqslant r<R+x_{I}$, the electroneutrality does not apply. By integrating Eq. A.10 between $R+x_{I}$ and $r$ the cation (and anion) concentration field is given by:

$$
c^{+}(r)=\frac{j_{g}}{z \mathrm{~F}}\left(\frac{R}{r}\right)^{2} \frac{1}{2 D_{c}}\left(\frac{R^{2}}{R+x_{I}}-\frac{R^{2}}{r}\right),
$$

where, $c^{+}\left(R+x_{I}\right)$ is neglected in front of $c^{+}(r)$ in this neutral region (as in Ref. 14). By combining Eqs. A.8 and A.10, the gradient of electric potential, in the neutral region, is given by:

$$
\frac{d \phi}{d r}=\frac{D_{a}}{z u_{a} \mathrm{~F}}\left(\frac{1}{\frac{r^{2}}{R+x_{I}}-r}\right) .
$$

By integrating this latter equation between $r\left(>R+x_{I}\right)$ and $L^{\prime}$, we obtain for the electric potential: 

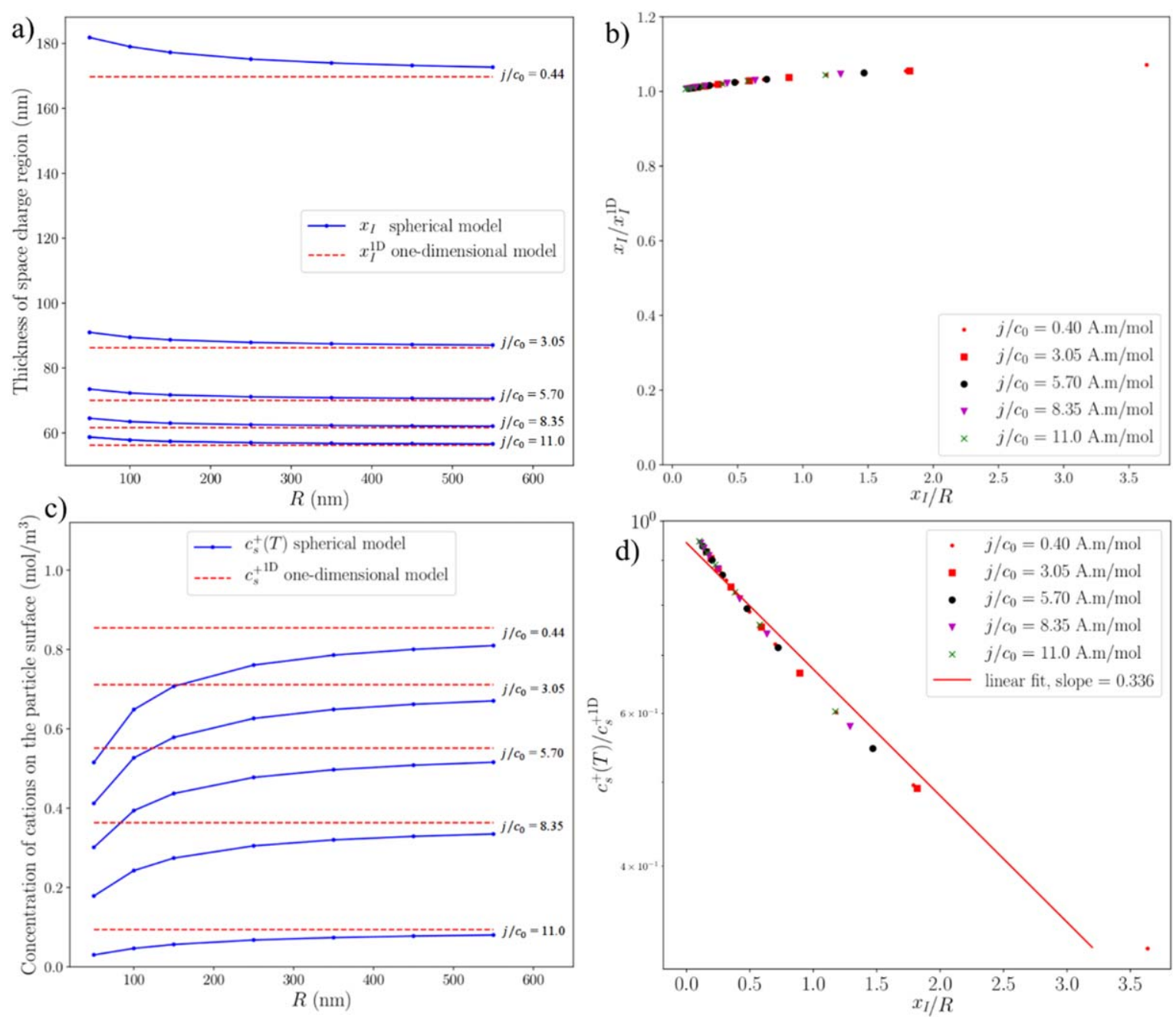

Figure A•1. Comparison between spherical and one-dimensional models for the prediction of both space charge region/layer thickness (a), (b) and cation surface concentration (c), (d). Space charge region thicknesses ( $x_{I}$ for the spherical model, $x_{I}{ }^{1 \mathrm{D}}$ for the one-dimensional model) (a) and the ratio $x_{I} / x_{I}{ }^{1 \mathrm{D}}(\mathrm{b})$ as a function of respectively the particle radius $R$ and the ratio $x_{I} / R$, for several values of the ratio $j / c_{0}$. Cation surface concentrations $\left(c_{s}^{+}(T)\right.$ for the spherical model, $c_{s}^{+1 \mathrm{D}}$ for the one-dimensional model) (c) and the ratio $c_{s}^{+}(T) / c_{s}^{+1 \mathrm{D}}$ (d) as a function of respectively the particle radius $R$ and the ratio $x_{I} / R$, for several values of $j / c_{0}$ (given in A.m mol ${ }^{-1}$ ).

$$
\phi(r)=\phi\left(L^{\prime}\right)-\frac{D_{a}}{z u_{a} \mathrm{~F}}\left[\log \left(\frac{L^{\prime}-\left(R+x_{I}\right)}{r-\left(R+x_{I}\right)}\right)-\log \left(\frac{L^{\prime}}{R+x_{I}}\right)\right] .
$$

Equation A.13 shows that $\phi\left(r \rightarrow R+x_{I}\right) \rightarrow-\infty$, and from Eq. A.9, $c^{-}\left(r \rightarrow R+x_{I}\right) \rightarrow 0$. Since $\phi(r)$ is expected to be a monotonic function, $c^{-} \rightarrow 0$ in the space charge region (see also concentration profiles obtained numerically ${ }^{14}$ ).

In the space charge region, Poisson equation becomes:

$$
\frac{1}{r^{2}} \frac{d}{d r}\left(r^{2} \frac{d \phi}{d r}\right)=-\frac{\mathrm{F}}{\varepsilon \varepsilon_{0}} z c^{+} .
$$

Because of the high electric field in the space charge region (Eq. A.8), the transport of cations is mainly due to migration and Eq. A.7 becomes:

$$
z u_{c} \mathrm{~F} c^{+} \frac{d \phi}{d r}=\frac{j_{g}}{z \mathrm{~F}}\left(\frac{R}{r}\right)^{2}
$$

Combining Eqs. A.14 and A-15, after integration between $r$ and $R+x_{I}$ (and considering $c^{+}\left(R+x_{I}\right) \gg c^{+}(r)$ ), leads to:

$$
c^{+}(r)=\left[\frac{2}{3} \frac{(z \mathrm{~F})^{3} u_{c}}{\varepsilon \varepsilon_{0} j_{g}}\left(\frac{\left(R+x_{I}\right)^{3}-r^{3}}{R^{2}}\right)\right]^{-1 / 2} .
$$

From Eq. A·15, after integration, the electric potential is given by:

$$
\phi(r)=\phi\left(x_{I}+R\right)-R \sqrt{\frac{2 j_{g}}{3 z \mathrm{~F} u_{c} \varepsilon \varepsilon_{0}}} \int_{r}^{x_{I}+R} \frac{\sqrt{\left(x_{I}+R\right)^{3}-x^{3}}}{x^{2}} d x .
$$

From the latter equation, introducing $\delta V=\phi\left(x_{I}+R\right)-\phi(R)$ the potential drop across the space charge, the thickness of the space charge layer $x_{I}$ can be deduced from:

$$
\delta V=R \sqrt{\frac{2 j_{g}}{3 z \mathrm{~F} u_{c} \varepsilon \varepsilon_{0}}} \int_{R}^{x_{I}+R} \frac{\sqrt{\left(x_{I}+R\right)^{3}-x^{3}}}{x^{2}} d x .
$$


The values obtained for $x_{I}$ and $c^{+}\left(R=R_{g}\right)=c_{s}^{+}(T)$, for this spherical case, are compared to the prediction of the one-dimensional model of Chazalviel (Eqs. 27 and 25 in Ref. 14]):

$$
\begin{gathered}
x_{I}{ }^{1 \mathrm{D}}=\left(\frac{3}{2}\right)^{2 / 3} \delta V^{2 / 3}\left(\frac{\varepsilon \varepsilon_{0} z \mathrm{~F} u_{c}}{2 j_{g}}\right)^{1 / 3}, \\
c_{s}^{+1 \mathrm{D}}=\left(\frac{\varepsilon \varepsilon_{0}}{2(z \mathrm{~F})^{3} u_{c}} \frac{j_{g}}{x_{I}^{1 \mathrm{D}}}\right)^{1 / 2} .
\end{gathered}
$$

$x_{I}$ is found to stay close to $x_{I}{ }^{1 \mathrm{D}}$ whatever the ratios $j / c_{0}$ and $x_{I} / R$ (for the ranges encountered here), Fig. A-1a. As expected, $x_{I} \rightarrow x_{I}{ }^{1 \mathrm{D}}$ for $x_{I} / R \rightarrow 0$, Fig. A.1b.

The same trend is obtained for $c_{s}^{+}(T) / c_{s}^{+1 \mathrm{D}}$ but $c_{s}^{+}(T)$ is significantly lower than $c_{s}^{+1 \mathrm{D}}$ for high values of $x_{I} / R$, Fig. A.1c. A useful observation is that the curves $c_{s}^{+}(T) / c_{s}^{+1 \mathrm{D}}$, as a function of $x_{I} / R$, coincide for all values of $j / c_{0}$, Fig. A. $1 \mathrm{~d}$. This enables a separation of the variables in the relation providing $c_{s}^{+}(T)$ for the spherical case:

$$
c_{s}^{+}(T)=f\left(x_{I} / R\right)\left[c_{s}^{+1 \mathrm{D}}\left(j, c_{0}\right)\right],
$$

where the indeterminate function $f\left(x_{I} / R\right)$ can be roughly considered as an exponential function, $f(x)=\exp (-0.336 x)$, Fig. A.1d.

\section{Appendix B. Estimation of the Potential Drop Across the Space Charge Region}

During a galvanostatic electrolysis, the cell voltage $\Delta V$ evolves as shown in Fig. B 1 a for the particular case $c_{0}=0.5 \mathrm{M}, j=133 \mathrm{~mA}$ $\mathrm{cm}^{-2}$. Just after the application of the current, there is a decrease of $\Delta V$ up to $\sim 5 \mathrm{~s}$. This initial variation is related to a capacitive effect on a rather large time scale compared to usual electrochemical situations $(\sim 1 \mathrm{~ms})$; de Bruyn explained this effect by the influence of natural convection. ${ }^{45}$

At the same time, an increasing part of the applied current (all the current from the moment when $\Delta V$ stops to decrease with $t$ ) is used to convert the cations into metal at the cathode surface (electrochemical reduction). Due to the non-renewal of the electrolyte (stagnant solution) there is a depletion region close to the initially flat cathode surface. At the surface of the cathode, the concentration tends towards $\sim 0$. The instant when the concentration reaches $\sim 0$ (Sand's time) marks the onset of branch growth. ${ }^{56}$ At the same time, cell voltage rises (partly because of the increase in the resistivity, itself induced by electrolyte depletion) up to a maximum value, Fig. B.1a. The time at the peak is close to Sand's time and it also marks the beginning of branch growth. ${ }^{45,56}$

Next, $\Delta V$ decreases linearly with time because the growth front (cathode) gets closer to the anode and the corresponding interelectrode distance decreases linearly with time (as shown and discussed in several previous works ${ }^{21,45}$ ). By considering that the branches are a perfect electrical conductor, $\Delta V$ is the sum of several contributions:

$$
\Delta V=|\eta|+\eta_{a}+\delta V^{\prime}+\delta V_{c o n c}+\delta V_{\text {ohm }},
$$

where:

- $\eta_{a}$ is the anodic overpotential

- $\delta V_{\text {ohm }}$ the potential drop where the concentration field is uniform (the enriched anodic region is not considered), ahead the region of concentration gradients (Fig. B.2)

- $\delta V_{\text {conc }}$ the potential drop, where there are concentration gradients and electroneutrality of the solution (typically on a length $L_{D}$ related to the average growth velocity by $L_{D}=D / v_{g}$ ), between the region with uniform concentration field and the region where the fields (concentration and potential) cannot be considered as onedimensional (due to the branch microstructure)

- $\delta V^{\prime}$ is the remaining potential drop across the space charge region and a small part of the neutral region, Fig. B.2.

By considering the system as one dimensional, from a distance $H$ $\left(\sim R_{g} \in[150,550 \mathrm{~nm}]\right)$ above the top of the branches (Fig. B 2$)$, the two last terms in the right hand side of Eq. B 1 are given by:

$$
\begin{gathered}
\delta V_{\text {conc }}=\int_{\bar{x}_{f}+H}^{\bar{x}_{f}+H+L_{D}} d \phi, \\
\delta V_{\text {ohm }}=\rho_{e}\left(c_{0}\right) j\left(L-\bar{x}_{f}-H-L_{D}\right),
\end{gathered}
$$

where $\bar{x}_{f}$ is the average location of the growth front (Fig. B.2) and $\rho_{e}\left(c_{0}\right)$ the electric resistivity of the solution. Assuming that the overpotentials are negligible compared to the other contributions (this is generally satisfied, and even comforted for fast kinetics as considered here, since the overpotentials fall in the range $\sim 10$ $100 \mathrm{mV}$ ) as well as $\delta V_{\text {conc }}$, Eq. B.1 takes the simplified form:

$$
\Delta V \approx \delta V^{\prime}+\delta V_{o h m}
$$

$\delta V_{\text {conc }}$ has been neglected compared to $\delta V_{\text {ohm }}$, as in previous works. $^{41,45}$ This can be justified because $\delta V_{\text {conc }}$ slowly diverges when the concentration at the growth front tends towards $\sim 0$. Indeed, in this region, the dependence of the electric potential on the local concentration can be deduced from the general expression of the electric current inside the electrolyte which is given here by $\left(c=c^{+}=c^{-}\right)$:

$$
j=z^{2} \mathrm{~F}^{2}\left(u_{a}+u_{c}\right) c(x) \frac{\partial \phi}{\partial x}+z \mathrm{~F}\left(D_{c}-D_{a}\right) \frac{\partial c}{\partial x} .
$$

This latter equation can be simplified by neglecting the diffusion term (this is reasonable, first because the diffusion coefficients are expected to be close, and second because this term does not contain diverging terms contrary to the migration term):

$$
j \approx z^{2} \mathrm{~F}^{2}\left(u_{a}+u_{c}\right) c(x) \frac{\partial \phi}{\partial x} .
$$

In this neutral region, the concentration profile satisfies the diffusion equation: ${ }^{24}$

$$
\frac{\partial c}{\partial t}=D \frac{\partial c}{\partial x^{2}}
$$

which can be converted to its stationary form using the coordinate relative to the front $x^{\prime}=x-v_{g} t$ :

$$
\frac{d^{2} c}{d x^{\prime 2}}+L_{D}^{-1} \frac{d c}{d x^{\prime}}=0
$$

A solution of this latter equation is:

$$
\frac{c\left(x^{\prime}\right)-c_{0}}{c^{*}-c_{0}}=\exp \left(-\frac{x^{\prime}-H}{L_{D}}\right),
$$

where $c^{*}$ is the electrolyte concentration at $x^{\prime}=H$ (Fig. B-2). By integrating Eq. B.6 between $\bar{x}_{f}+H$ and $\bar{x}_{f}+H+L_{D}$, using Eq. B.9, we obtain:

$$
\delta V_{\text {conc }}=\frac{j}{z^{2} \mathrm{~F}^{2}\left(u_{a}+u_{c}\right)} \frac{L_{D}}{c_{0}} \log \left(1+(\exp (1)-1) \frac{c_{0}}{c^{*}}\right) .
$$

Since $L_{D}=D / v_{g}$ and $v_{g} \propto j / c_{0}$ (Eq. 2), according to Eq. B.10, $\delta V_{\text {conc }}$ does not directly depend on $j$ nor $c_{0}$. Eq. B.10 shows that the 



Figure B·1. (a) The cell voltage $\Delta V$ as a function of time for $c_{0}=0.5 \mathrm{M}$ and $=133 \mathrm{~mA} \mathrm{~cm}{ }^{-2}$, the red line (corresponding to the linear fit of the decreasing part of the curve on the right of the peak) highlights the linear decrease of $\Delta V$ with $t$. (b) The estimated potential drop across the space charge region as a function of the applied current density $j$ and for several concentrations $c_{0}$.

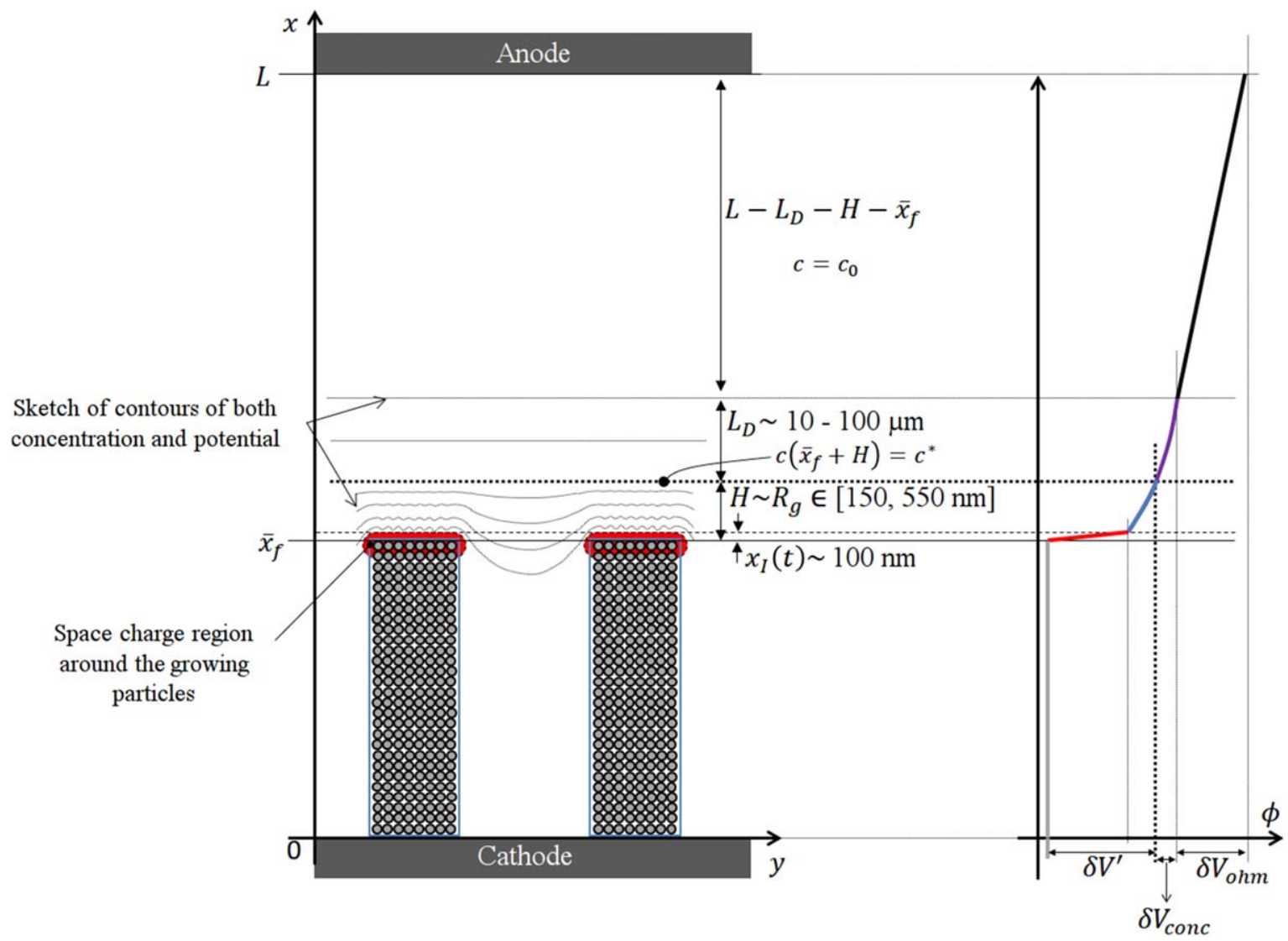

Figure B·2. Sketch of the electric potential profile across the electrochemical cell.

divergence of $\delta V_{\text {conc }}$ with $1 / c^{*}$ is slight because of the logarithmic dependence. The value of the electrolyte depletion $c_{0} / c^{*}$, and its trend with both $j$ and $c_{0}$, can be estimated by taking the value of $c^{+}(R)=c_{s}^{+}(T)$, given by Eq. A.20, for $c^{*}\left(=c\left(\bar{x}_{f}+H\right)\right)$; note that $c_{0} / c^{*}$ and $\delta V_{\text {conc }}$ are therefore overestimated. We find that $\delta V_{\text {conc }}$ decreases with $j$ and for the worst case $\left(0.75 \mathrm{M}, j=33 \mathrm{~mA} \mathrm{~cm}^{-2}\right)$, $\delta V_{\text {conc }}<0.093 \mathrm{~V}$. This rather low upper value, and the fact that $\delta V_{\text {conc }}$ decreases with $j$, (see below and Fig. B $\cdot 1 \mathrm{~b}$ ) allows this term to be neglected.

Considering a cell length $L$ well higher than $L_{D}$ and $\bar{x}_{f}$ (and $x_{I}$ ), $\delta V_{\text {ohm }} \approx \rho_{e}\left(c_{0}\right) j L$, and its value can be estimated from the value of $\Delta V$ just after the initial capacitive phase (for $t<\sim 5 \mathrm{~s}$ in Fig. B.1a) when the concentration profile has not changed much yet (this value is taken as the minimum value on the plot $\Delta V$ as a function of time). 
By subtracting this last value from the maximum value of $\Delta V$ (at the peak), we measure $\delta V^{\prime} . \delta V^{\prime}$ thus determined actually correspond to the actual space charge region at the initially flat cathode surface $\left(\delta V^{\prime}=\right.$ potential drop across the space charge region for a onedimensional system for which $H=0$ ). Since, after the peak, there is no discontinuity of the cell voltage and that it decreases linearly with time, this suggests that $\delta V^{\prime}$ remains almost the same even if the electroactive surface changes significantly (flat surface $\rightarrow$ top of the ramified branches, $H$ can no longer be considered as 0 ). We deduce that this estimation of $\delta V^{\prime}$ gives access to $\delta V$.

Overall, we obtain values for $\delta V$ of about $1 \mathrm{~V}$. The same value has already been reported for the same system. ${ }^{41}$ As already observed in the work of de Bruyn $1997,{ }^{45}$ we observe a slow decrease in $\delta V$ with the applied current density $j$ (note that this trend is not expected to be due to not taking into account $\delta V_{\text {conc }}$ since this latter would induce the opposite trend as discussed just above).

\section{ORCID}

Fabien Chauvet (iD https://orcid.org/0000-0002-3102-0368

\section{References}

1. K. Nishikawa, T. Mori, T. Nishida, Y. Fukunaka, M. Rosso, and T. Homma, "In situ observation of dendrite growth of electrodeposited li metal." J. Electrochem. Soc., 157, A1212 (2010).

2. A. Maraschky and R. Akolkar, "Mechanism explaining the onset time of dendritic lithium electrodeposition via considerations of the $\mathrm{Li}+$ transport within the solid electrolyte interphase." J. Electrochem. Soc., 165, D696 (2018).

3. P. Bai, J. Li, F. R. Brushett, and M. Z. Bazant, "Transition of lithium growth mechanisms in liquid electrolytes." Energy Environ. Sci., 9, 3221 (2016)

4. M. Paunovic and M. Schlesinger, Fundamentals of Electrochemical Deposition (Wiley, Hoboken, New Jersey, NJ) 2nd ed. (2006).

5. N. T. Nesbitt, M. Ma, B. J. Trześniewski, S. Jaszewski, F. Tafti, M. J. Burns, W. A. Smith, and M. J. Naughton, "Au dendrite electrocatalysts for $\mathrm{CO}_{2}$ electrolysis." The Journal of Physical Chemistry C, 122, 10006 (2018).

6. J. J. Burk and S. K. Buratto, "Electrodeposition of Pt nanoparticle catalysts from $\mathrm{H}_{2} \mathrm{Pt}(\mathrm{OH})_{6}$ and their application in PEM fuel cells." The Journal of Physical Chemistry C, 117, 18957 (2013).

7. H. Chen, P. Kannan, L. Guo, H. Chen, and D.-H. Kim, "Direct growth of highly branched crystalline au nanostructures on an electrode surface: their surface enhanced raman scattering and electrocatalytic applications." J. Mater. Chem., 21, 18271 (2011)

8. D. Xu, X. Yan, P. Diao, and P. Yin, "Electrodeposition of vertically aligned palladium nanoneedles and their application as active substrates for surface-enhanced raman scattering." The Journal of Physical Chemistry C, 118, 9758 (2014).

9. W. Ye, J. Yan, Q. Ye, and F. Zhou, "Template-free and direct electrochemical deposition of hierarchical dendritic gold microstructures: growth and their multiple applications." The Journal of Physical Chemistry C, 114, 15617 (2010).

10. Y. Li, W.-Z. Jia, Y.-Y. Song, and X.-H. Xia, "Superhydrophobicity of 3d porous copper films prepared using the hydrogen bubble dynamic template." Chem. Mater., 19, 5758 (2007)

11. A. Iranzo, F. Chauvet, and T. Tzedakis, "Synthesis of submicrometric dendritic iron particles in an electrochemical and vibrating hele-shaw cell: study of the growth of ramified branches." Electrochim. Acta, 250, 348 (2017).

12. G. González, M. Rosso, and E. Chassaing, "Transition between two dendritic growth mechanisms in electrodeposition." Phys. Rev. E, 78, 011601 (2008).

13. V. Fleury, J.-N. Chazalviel, M. Rosso, and B. Sapoval, "The role of the anions in the growth speed of fractal electrodeposits." J. Electroanal. Chem. Interfacial Electrochem., 290, 249 (1990).

14. J.-N. Chazalviel, "Electrochemical aspects of the generation of ramifiedmetallic electrodeposits." Phys. Rev. A, 42, 7355 (1990).

15. C. Léger, J. Elezgaray, and F. Argoul, "Internal structure of dense electrodeposits." Phys. Rev. E, 61, 5452 (2000).

16. M. Matsushita, M. Sano, Y. Hayakawa, H. Honjo, and Y. Sawada, "Fractal structures of zinc metal leaves grown by electrodeposition." Phys. Rev. Lett., 53, 286 (1984).

17. D. Grier, E. Ben-Jacob, R. Clarke, and L. M. Sander, "Morphology and microstructure in electrochemical deposition of zinc." Phys. Rev. Lett., 56, 1264 (1986).

18. Y. Sawada, A. Dougherty, and J. P. Gollub, "Dendritic and fractal patterns in electrolytic metal deposits." Phys. Rev. Lett., 56, 1260 (1986).

19. E. Ben-Jacob and P. Garik, "The formation of patterns in non-equilibrium growth." Nature, 343, 523 (1990).

20. E. Ben-Jacob, G. Deutscher, P. Garik, N. D. Goldenfeld, and Y. Lareah, "Formation of a dense branching morphology in interfacial growth." Phys. Rev. Lett., 57, 1903 (1986).

21. V. Fleury, M. Rosso, J.-N. Chazalviel, and B. Sapoval, "Experimental aspects of dense morphology in copper electrodeposition.” Phys. Rev. A, 44, 6693 (1991).
22. J. M. Huth, H. L. Swinney, W. D. McCormick, A. Kuhn, and F. Argoul, "Role of convection in thin-layer electrodeposition." Phys. Rev. E, 51, 3444 (1995).

23. G. Marshall and P. Mocskos, "Growth model for ramified electrochemical deposition in the presence of diffusion, migration, and electroconvection." Phys. Rev. E, 55, 549 (1997).

24. J. Newman and K. Thomas-Alyea, Electrochemical Systems (Wiley, New York NY) (2012).

25. C. Léger, J. Elezgaray, and F. Argoul, "Experimental demonstration of diffusionlimited dynamics in electrodeposition." Phys. Rev. Lett., 78, 5010 (1997).

26. F. Argoul, A. Arneodo, G. Grasseau, and H. L. Swinney, "Self-similarity of diffusion-limited aggregates and electrodeposition clusters." Phys. Rev. Lett., 61, 2558 (1988).

27. F. Sagués, M. Q. López-Salvans, and J. Claret, "Growth and forms in quasi-twodimensional electrocrystallization." Phys. Rep., 337, 97 (2000).

28. C. Léger, L. Servant, J. L. Bruneel, and F. Argoul, "Growth patterns in electrodeposition." Physica A, 263, 305 (1999).

29. T. A. Witten and L. M. Sander, "Diffusion-limited aggregation." Phys. Rev. B, 27, 5686 (1983).

30. R. L. Smith and S. D. Collins, "Generalized model for the diffusion-limited aggregation and eden models of cluster growth." Phys. Rev. A, 39, 5409 (1989).

31. B. Sapoval, M. Rosso, and J.-F. Gouyet, "The fractal nature of a diffusion front and the relation to percolation." J. Physique Lett., 46, 149 (1985).

32. S. Miyashita, Y. Saito, and M. Uwaha, "Fractal aggregation growth and the surrounding diffusion field." J. Cryst. Growth, 283, 533 (2005).

33. K. Nishikawa, Y. Fukunaka, E. Chassaing, and M. Rosso, "Electrodeposition of metals in microgravity conditions." Electrochim. Acta, 100, 342 (2013).

34. V. Fleury, J.-N. Chazalviel, and M. Rosso, "Theory and experimental evidence of electroconvection around electrochemical deposits." Phys. Rev. Lett., 68, 2492 (1992).

35. D. G. Grier, D. A. Kessler, and L. M. Sander, "Stability of the dense radial morphology in diffusive pattern formation." Phys. Rev. Lett., 59, 2315 (1987).

36. J. L. Barton, J. O. Bockris, and A. R. J. P. Ubbelohde, "The electrolytic growth of dendrites from ionic solutions." Proceedings of the Royal Society of London. Series A. Mathematical and Physical Sciences 268, 485 (1962).

37. Y. Oren and U. Landau, "Growth of zinc dendrites in acidic zinc chloride solutions." Electrochim. Acta, 27, 739 (1982).

38. R. Akolkar, "Mathematical model of the dendritic growth during lithium electrodeposition." J. Power Sources, 232, 23 (2013).

39. V. Fleury, "Branched fractal patterns in non-equilibrium electrochemical deposition from oscillatory nucleation and growth." Nature, 390, 145 (1997).

40. V. Fleury, J. Kaufman, and B. Hibbert, "Evolution of the space-charge layer during electrochemical deposition with convection." Phys. Rev. E, 48, 3831 (1993).

41. V. Fleury, J.-N. Chazalviel, and M. Rosso, "Coupling of drift, diffusion, and electroconvection, in the vicinity of growing electrodeposits." Phys. Rev. E, $\mathbf{4 8}$ 1279 (1993).

42. W. W. Mullins and R. F. Sekerka, "Morphological stability of a particle growing by diffusion or heat flow." J. Appl. Phys., 34, 323 (1963).

43. T. Devers, I. Kante, L. Allam, and V. Fleury, "Preparation of dendritic tin nanoaggregates by electrodeposition." J. Non-Cryst. Solids, 321, 73 (2003).

44. I. Kante, P. Andreazza, C. Andreazza, T. Devers, L. Allam, and V. Fleury, "Structure and atoms mixing in electrodeposited sn(au) nanoaggregates." J. Cryst. Growth, 277, 599 (2005).

45. J. R. de Bruyn, "Physical and electrochemical contributions to the cell voltage in the thin-layer electrochemical deposition of copper at constant current." Phys. Rev. E 56, 3326 (1997).

46. M. Uwaha and Y. Saito, "Aggregation growth in a gas of finite density: velocity selection via fractal dimension of diffusion-limited aggregation." Phys. Rev. A, $\mathbf{4 0}$ 4716 (1989).

47. J. S. Langer, "Instabilities and pattern formation in crystal growth." Rev. Mod. Phys., 52, 1 (1980).

48. M. E. Glicksman, Principles of Solidification (Springer, New York, NY) (2011).

49. A. A. Golovin, "Interfacial phenomena in materials science, in pattern formation at interfaces." ed. P. Colinet and A. Nepomnyashchy (Springer, Berlin, Wien) (2010).

50. A. A. Golovin and S. H. Davis, "Effect of anisotropy on morphological instability in the freezing of a hypercooled melt." Physica D, 116, 363 (1998).

51. L.-G. Sundström and F. H. Bark, "On morphological instability during electrodeposition with a stagnant binary electrolyte." Electrochim. Acta, 40, 599 (1995).

52. C. P. Nielsen and H. Bruus, "Morphological instability during steady electrodeposition at overlimiting currents." Phys. Rev. E, 92, 052310 (2015).

53. A. J. Bard and L. R. Faulkner, Electrochemical Methods Fundamentals and Applications (Wiley, New York, NY) 2nd ed. (2001).

54. Y. D. Gamburg and G. Zangari, Theory and Practice of Metal Electrodeposition (Springer, Berlin) (2011).

55. A. Iranzo and F. Chauvet, "Synthesis of in situ purified iron nanoparticles in an electrochemical and vibrating microreactor: study of ramified branch fragmentation by oscillating bubbles." Microfluid. Nanofluid., 23, 45 (2019).

56. F. Argoul, E. Freysz, A. Kuhn, C. Léger, and L. Potin, "Interferometric characterization of growth dynamics during dendritic electrodeposition of zinc." Phys. Rev. E, 53, 1777 (1996). 